\title{
Acetate metabolic requirement of avian pathogenic Escherichia coli promotes its intracellular proliferation within macrophage
}

Xiangkai Zhuge ${ }^{1,2,3,4,5}$, Yu Sun 1,2, Min Jiang 1,2, Juanfang Wang ${ }^{1,2}$, Fang Tang ${ }^{1,2}$, Feng Xue ${ }^{1,2}$, Jianluan Ren ${ }^{1,2}$, Weiyun Zhu ${ }^{5}$ and Jianjun Dai ${ }^{1,2,3,4^{*}}$ (D)

\begin{abstract}
Avian pathogenic Escherichia coli (APEC) is a facultative intracellular pathogen, and intracellular persistence in macrophages is essential for APEC extraintestinal dissemination. Until now, there is still no systematic interpretation of APEC intracellular proliferation. Intracellular survival factors, especially involved in pathometabolism, need to be further revealed. Acetate plays critical roles in supporting energy homeostasis and acts as a metabolic signal in the inflammatory response of eukaryotes. In this study, we identified that APEC acs-yjcH-actP operon, encoding acetate assimilation system, presented the host-induced transcription during its proliferation in macrophages. Our result showed that this acetate assimilation system acted as a novel intracellular survival factor to promote APEC replication within macrophages. Furthermore, deletion of acs-yjcH-actP operon in APEC decreased its cytotoxic level to macrophages. qRT-PCR results showed that the production of pro-inflammatory cytokines (IL-1 $\beta, I L-6$, IL-8, IL-12 3 , and TNF-a) and iNOS in FY26 $\triangle a c s-y j c H$-actP infected macrophages were obviously down-regulated compared to that in wild-type FY26 infected cells. Deletion of actP/yjcH/acs genes attenuated APEC virulence and colonization capability in avian lungs in vivo for colibacillosis infection models. And acetate assimilation system acted as a virulence factor and conferred a fitness advantage during APEC early colonization. Taken together, our research unravelled the metabolic requirement of APEC intracellular survival/replication within macrophages, and acetate metabolic requirement acted as an important strategy of APEC pathometabolism. The intracellular acetate consumption during facultative intracellular bacteria replication within macrophages promoted immunomodulatory disorders, resulting in excessively pro-inflammatory responses of host macrophages.
\end{abstract}

\section{Introduction}

The intracellular infection of pathogenic bacteria causes metabolic adaptation responses to exploit nutrient or energy source of host cells, aiming to enhance the active survival and proliferation within host intracellular compartments [1-3]. The growing evidences show the pathogenic bacteria can regulate the expression of its metabolic pathways or virulence factors to deprive the necessary nutrients of the host cells, accompanied

\footnotetext{
*Correspondence: daijianjun@njau.edu.cn

${ }^{1}$ MOE Joint International Research Laboratory of Animal Health and Food Safety, College of Veterinary Medicine, Nanjing Agricultural University, Nanjing, China

Full list of author information is available at the end of the article
}

by host metabolic disorders as well as dysregulated antimicrobial reactions $[2,4,5]$. Such adaptations lead to changes in the metabolism between internalized bacteria and host cells, and the final outcome links with the pathogenicity $[4,6,7]$. Therefore, the phenomenon that the complex metabolic interactions during bacterial intracellular infection is termed as "pathometabolism" [1]. Pathometabolism has gradually become a research hotspot, and researchers attempt to reveal critical intracellular metabolic pathways and achieve a deeper understanding of bacterial infection $[4,7]$.

The intracellular bacteria can successfully survive and replicate within phagocytosis or non-phagocytosis cells, and also grow extracellularly in host body fluid environment or laboratory culture conditions [8]. Typical

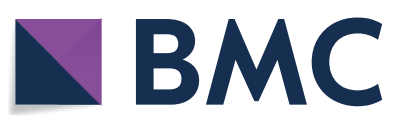

(c) The Author(s) 2019. This article is distributed under the terms of the Creative Commons Attribution 4.0 International License (http://creativecommons.org/licenses/by/4.0/), which permits unrestricted use, distribution, and reproduction in any medium, provided you give appropriate credit to the original author(s) and the source, provide a link to the Creative Commons license, and indicate if changes were made. The Creative Commons Public Domain Dedication waiver (http://creativecommons.org/ publicdomain/zero/1.0/) applies to the data made available in this article, unless otherwise stated. 
facultative intracellular bacteria, including Mycobacterium tuberculosis, Salmonella typhi, Brucella, Legionella pneumophila, and Yersinia pestis, replicate in specific bacteria-containing vacuoles (PCVs) within infected host cells [1]. The other pathogens, including Shigella, Listeria, Group A Streptococcus, and Neisseria meningitides, can escape from phagolysosomes into the cytosol of infected host cells where intracellular bacteria efficiently replicate $[1,9]$. The cytosolic host nutrients can be directly acquired by the cytosolically replicating pathogens, whereas the intracellular bacteria residing in PCVs obtain the nutrients by transporters located in vacuolar membranes $[1,10,11]$. It seems that there is an intimate coordination between the metabolic systems and so-called "intracellular survival factors" of the intracellular pathogens, and the relationship is critical for the various stages of intracellular infection, such as replication within host cell compartments and reinfection of new host cells [12, 13]. Recent reports reveal that the metabolic activities of intracellular bacteria are essential for its resistance to the antimicrobial defense of host cells. Current research is still far from clear elucidation of the bacterial metabolism during its intracellular infection.

Macrophages play critical roles in host defense responses against microbial pathogens and act as the front line of host cells to encounter the pathogenic bacteria [4]. The successful pathogen must develop several mechanisms to hijack and overcome (at least partially) these antimicrobial responses $[14,15]$. Moreover, it is not surprising that intracellular bacteria exploit and manipulate host nutrient metabolism as part of their intracellular survival and replication strategies [16]. Many studies show that intracellular replication of bacteria is closely linked to lipid metabolism of macrophages and perturbations of lipid metabolism, such as fatty acids and cholesterol, to prevent immune defenses and cellular functions of the infected macrophages $[4,7]$.

Acetate is one of the metabolic end products for glucose metabolism in enterobacteria. When growing aerobically, glucose is assimilated and first conversed into pyruvate through the glycolytic pathway, and the pyruvate is further transferred to acetyl-CoA by oxidative decarboxylation [17]. Then acetyl-CoA, so-called activated acetate, is conversed into acetate or acetic acid through acetyl-phosphate, including two-step reactions via phosphotransacetylase (Pta) and acetate kinase (AckA), encoded by E. coli ackA-pta operon [18]. In turn, acetate can be used as the sole carbon source of E. coli and other microbiota [17-20]. Under low concentration in the natural environment, acetate can be recruited by $E$. coli via specific membrane transporters. Two membrane transporters in E. coli are closely connected to trans-membrane transportation of acetate.
The actP gene, variously named $y j c G$, encodes an acetate permease [20]. The satP gene in E. coli encodes a succinate-acetate transporter [21]. Single deletion of satP or actP genes in $E$. coli leads to a partial decrease in acetate uptake while double deletion of satP and act $P$ genes almost abolishes $E$. coli acetate uptake activity [17, 21]. The actP, yjcH (encoding a hypothetical membrane protein), and acs (encoding acetyl-CoA synthetase, Acs) genes comprise one operon, highly conserved in E. coli. Bacteria can use high-affinity Acs to assimilate acetate as a carbon source under low concentrations. Acs catalyzes the conversion of intracellular acetate to acetyl-CoA by an irreversible reaction in $E$. coli, and acetyl-CoA enters central metabolism, such as TCA and glyoxylate cycle $[17,18]$.

It is well-known that $E$. coli lives in the intestine of warm-blooded animals as a harmless commensal strain. Due to loss or gain of mobile virulence-related islands and genetic elements, E. coli evolves into two major pathogenic categories: IPEC (Intestinal pathogenic E. coli) and ExPEC (Extra-intestinal pathogenic E. coli) [22-24]. IPEC harbors a series of intestinal virulence factors to cause intestinal and diarrheal diseases [24]. However, ExPEC holds an enhanced capability to cause infection in specific extraintestinal niches, such as colonizing in the human urinary tract, avian respiratory system, the bloodstream, and central nervous system [22, 25, 26]. Based on the different hosts and syndromes, ExPEC pathotypes are designated as avian pathogenic E. coli (APEC), uropathogenic E. coli, sepsis-associated E. coli (SEPEC), and neonatal meningitis E. coli (NMEC). In recent years, researchers have gradually recognized that $\mathrm{APEC} / \mathrm{ExPEC}$ is a primary pathogen and acts as a major cause of the high-incidence of bacterial diseases [27-29]. APEC/ExPEC can evade the host immune barrier and escape from macrophage clearance into extraintestinal niches. APEC/ExPEC can utilize a variety of intracellular survival factors to successfully persist and replicate within infected phagocytosis cells [13]. Our previous studies indicate that ColV plasmids undertake critical function with APEC survival in macrophages. The twocomponent system PhoP/PhoQ controls HlyF (the ColV plasmid-encoded protein) to facilitate APEC escaping from phagolysosomes into the cytosol of infected macrophages [30].

The intracellular metabolic strategies utilized by APEC to enhance proliferative capacity within host phagocytosis cells remain poorly understood. In this study, owing to the comprehensive analysis of APEC acetate metabolic requirement, we revealed new identification of APEC pathogenic mechanism to promote its intracellular proliferation. Our results provided novel insights into metabolic crosstalk between pathogens and macrophages. 


\section{Materials and methods}

\section{Strains and plasmids construction}

The description of the strains, plasmids, and PCR primers was shown in Additional file 1 and Additional file 2. The highly virulent APEC strains (FY26 and CVCC249) and human ExPEC strain RS218 acted as typical models to identify the molecular pathogenesis of APEC/ExPEC $[30,31]$. The construction of the single acs, actP, satP, and acs-yjcH-actP operon mutants in wild-type FY26 was based on the Red recombinase method as the previously described [32]. The acs-yjcH-actP operon or the satP gene (containing the predicted promoters) was ligated in pSTV28 (a medium-copy plasmid, TaKaRa) to construct the complemented plasmid [30]. The complemented strains FY26Cacs-yjcH-actP and FY26CsatP were constructed by electroporating the complemented plasmids into the mutants, respectively.

\section{Cell culture}

HD11 cells, Chicken macrophage-like line, were cultured with RPMI 1640 media (supplemented with 10\% heat-inactivated fetal bovine serum, FBS) in a humidified atmosphere for $41{ }^{\circ} \mathrm{C}$ and $5 \% \mathrm{CO}_{2}$. The RAW264.7 cells were cultured with DMEM media (10\% FBS) at a $37{ }^{\circ} \mathrm{C}$ in a humidified incubator of $5 \% \mathrm{CO}_{2}$. DF-1 cells, the chicken embryo fibroblast cell line, were cultured with DMEM media (10\% FBS) at a $41{ }^{\circ} \mathrm{C}$ in a humidified incubator of $5 \% \mathrm{CO}_{2}$. The mixed cancer HEp2 cells were maintained with DMEM media (10\% FBS) at a $37{ }^{\circ} \mathrm{C}$ in a humidified incubator of $5 \% \mathrm{CO}_{2}$.

\section{RNA isolation and quantitative real-time reverse transcription PCR}

The total RNA was extracted from bacteria cultured in Luria-Bertani (LB) and isolated from blood for infected duck using an E.Z.N.A. bacterial RNA kit (Omega BioTek, Beijing, China) according to the manufacturer's protocol. The total RNA was treated with DNase I (Vazyme Biotech) for $1 \mathrm{~h}$ to remove the genomic DNA. To confirm free from contaminating DNA, PCR was conducted by the templates for RNA samples without reverse transcription.

For the total bacterial RNA extracted from the infected cells during bacteria infecting macrophages (HD11 and RAW246.7) and non-phagocytic cells, the monolayer cells were incubated with bacteria at an infection ratio 1:200 with $1 \mathrm{~h}$, and the infected cells were treated with antibiotic [13]. The cells were harvested at $4 \mathrm{hpi}$ or $8 \mathrm{hpi}$, and the cells were washed with PBS for three times. Then TRIZOL (invitrogen) was added to wells to lyse cells and extract the total RNA from the infected cells. In order to enrich bacterial mRNA, the total RNA from the infected cells was treated with MICROBEnrich ${ }^{\mathrm{TM}}$ Kit (Ambion; catalog no. AM1901) to remove host RNA [33, 34], and then processed with MICROBExpress ${ }^{\mathrm{TM}}$ (Ambion; catalog no. AM1905) to deplete the bacterial rRNA [33, 34]. The each sample was repeated the above processes. The realtime PCR was conducted as previously described [33], and the primers for qRT-PCR were shown in Additional file 2. qRT-PCR data from three individual experiments were used to measure the differences (fold-change) of these genes transcription. The transcription level of the housekeeping gene $d n a E$ acted as a reference to determine the expression level of targeted genes with the $\Delta \Delta C_{T}$ method as the previously described [35, 36]. qRTPCR was conducted with the AceQ qPCR SYBR Green Master Mix (Vazyme Biotech) according to the manufacturer's instruction.

Transcriptional level of selected genes for iNOS, proinflammatory cytokines (IL-1 $\beta$, IL-6, IL-8, IL-12 $\beta$, and TNF- $\alpha$ ), and anti-inflammatory cytokines (IL-4, IL-10, and IL-13) were quantified [15, 37-39], and the corresponding primers were showed in Additional file 2. HD11 cells infected with APEC/ExPEC strains were extracted using TRIZol ${ }^{\circledR}$ reagent (Invitrogen) at 4 hpi or 8 hpi. qRTPCR was performed by SYBR ${ }^{\circledR}$ PremixEx Taq $^{\text {TM }}$ (TaKaRa) to determine mRNA transcription of these cytokines. The gene transcription level was normalized to the gene $\beta$-actin with the $\Delta \Delta C_{T}$ method $[15,37,38]$.

\section{Growth curve}

For the growth experiments in Luria-Bertani (LB) broth, wild-type FY26, mutants, and complemented strains were cultured over a course of $12 \mathrm{~h}$ at $37^{\circ} \mathrm{C}$, starting at $1.0 \times 10^{7} \mathrm{CFU} / \mathrm{mL}$. The cultured $(200 \mu \mathrm{L})$ for $1 \mathrm{~h}$ apart were dropped to a 96-well plate in triplicate. A microplate reader was used to monitor the bacterial growth at an optical density $\mathrm{OD}_{600}$. The growth curve for each strain was obtained by averaged data from each read. The growth experiments in M9 minimal medium (carbon-source free) or plus acetic acid $(60 \mathrm{mM}, \mathrm{pH}$ 6) were performed according to the previously described [21]. $E$. coli strains were cultured over a course of $36 \mathrm{~h}$ at $37{ }^{\circ} \mathrm{C}$, starting at $5.0 \times 10^{7} \mathrm{CFU} / \mathrm{mL}\left(\mathrm{OD}_{600}=0.1\right)$. Growth curve assay was performed at least three individual experiments.

\section{Cytokines ELISA assay}

The monolayer cells were incubated with bacteria at a multiplicity of infection (MOI) of 10 with $1 \mathrm{~h}$. Then the supernatant of infected cells with three replicate wells was harvested at $8 \mathrm{hpi}$ and $16 \mathrm{hpi}$. The concentrations of cytokines IL- $1 \beta$, IL- 6 , IL- 8 , IL- $12 \beta$ and TNF- $\alpha$ in the supernatant of RAW264.7 cells were measured using the corresponding cytokine ELISA kits, including mouse IL-1 $\beta$ ELISA Kit (Abcam, ab197742), mouse IL-6 ELISA 
Kit (Abcam, ab100712), mouse IL-8 ELISA Kit (Sangon Biotech, C507010), mouse IL-12 $\beta$ ELISA Kit (Abcam, ab236717), and mouse TNF- $\alpha$ ELISA Kit (Abcam, ab208348). The cytokines ELISA assays were performed according to manufacturer's instructions provided by each ELISA kit. Data acquired from at least four individual experiments, and each assay was performed by three biological repetitions.

\section{Nitric oxide (NO) measurement}

The accumulation of nitrite in the cell supernatant acted as an indicator of NO production. NO production could be estimated by determining nitrite concentration, measured with Griess reaction [38]. The Griess reagent included $0.1 \% \mathrm{~N}$-(1-naphthyl)ethylenediamine dihydrochloride, $1 \%$ sulfanilamide, and $2.5 \% \mathrm{H}_{3} \mathrm{PO}$. The monolayer cells were incubated with bacteria at an infection ratio 1:10 with $1 \mathrm{~h}$. Then the supernatant of infected cells was harvested at $8 \mathrm{hpi}$. Equal volumes of Griess reagent and cell supernatant were mixed and incubated for $10 \mathrm{~min}$ at room temperature. A microplate reader (Biotek, USA) was used to determine the absorbance at $540 \mathrm{~nm}$. Data acquired from at least four individual experiments, and each assay was performed by three biological repetitions. The nitrite concentration of cell supernatant was determined by sodium nitrite standard curve.

\section{Cells infection assays}

Replication rates during wild-type FY26 and its variants were measured according to the described previously [40-42]. Briefly, the monolayer HD11 cells with three replicate wells were infected with each bacteria at a MOI of 10 . After $1 \mathrm{~h}$ of infection, the bacteria in the cell supernatant were discarded, and the infected macrophages were incubated with gentamicin to kill the non-internal bacteria. After incubation with gentamicin at six-time points $(2,4,6,8,10$, and $16 \mathrm{hpi})$, the infected cells were washed with PBS and lysed using the $0.1 \%$ Triton X-100. The plate counting for the total number of internalized bacteria was carried out with the serially diluted cell suspension. The internalized bacteria at $2 \mathrm{hpi}$ acted as the initial number of intracellular bacteria to determine the replication. Intracellular survival was measured by the bacterial number at different time points relative to initially internalized bacteria. These experiments were performed in triplicate with three biological repetitions.

\section{Immunofluorescent imaging assays}

Immunofluorescent imaging assays were conducted to quantify the number of intracellular bacteria according to the previous description [30,43]. Briefly, the monolayer HD11 cells were infected with wild-type FY26 and its variants at a MOI of 5 . The infected cells at $4 \mathrm{hpi}$ were washed twice with PBS, and fixed in 3\% paraformaldehyde for $15 \mathrm{~min}$. After incubation with $0.1 \%$ Triton X-100, the cells were treated with 5\% BSA. Cells were incubated with a polyclonal rabbit anti-APEC O2:K1 serum, which was prepared with the APEC strain DE205B, its background (O2:K1; ST140; Phylogroup B2; isolated from duck), as the previous described [30, 33, 44]. Then the infected cells were treated with FITC goat anti-rabbit IgG (EarthOx, San Francisco, USA), DAPI, and Phalloidin (actin staining; TRITC conjugated) as the described above. Immunofluorescent imaging of bacteria was detected using a Zeiss LSM-510 META confocal laser scanning microscope. The number of bacteria within infected HD11 cells could be directly counted from immunofluorescent imaging. Data for quantification of the intracellular bacteria represented the average proliferation level of at least 100 infected cells. These experiments were performed in triplicate with three biological repetitions.

\section{Animal experiments}

Ethics statement: All animal experimental protocols were handled according to the guidelines of Experimental Animal Management Measures of Jiangsu Province and were approved by the Laboratory Animal Monitoring Committee of Jiangsu Province, China.

The 7-day-old HBK-Q-SPF ducks for duck model were used to determine the effect of acs-yjcH-actP operon loss on APEC virulence as the previously described [45]. The duck groups (20 ducks for each group) were challenged intratracheally with bacteria at $5.0 \times 10^{5} \mathrm{CFU} /$ duck (dose/duck similar to $\mathrm{LD}_{50}$ of wild-type FY26). The mortality was calculated at the 7th day after post-infection, and the survival/mortality rates of eight groups were assessed. These experiments were performed in triplicate.

To measure the effect of acs-yjcH-actP operon loss on APEC colonization in vivo, the systemic infection experiment of duck model was conducted to assess the bacteria proliferation in duck lungs and blood [45]. The duck groups were challenged intratracheally with bacteria at $2.0 \times 10^{6} \mathrm{CFU} /$ duck (dose/duck similar to $\mathrm{LD}_{90}$ of wild-type FY26). 15 ducks for each group were euthanized and dissected at $24 \mathrm{hpi}$ to conduct systemic infection experiment. The number of bacteria colonizing in duckling tissues (lungs, spleen, liver, brain, kidney) and level of bacteremia in the blood were determined as follows: organ samples and blood were obtained from infected ducks; samples were weighed, suspended in PBS $(1 \mathrm{~mL} / \mathrm{g})$, and homogenized; the blood and homogenized tissues were serially diluted and plated on LB agar plates for counting. 
The 10-day-old SPF chicks (QYH Biotech) for chick colisepticemia model were used to determine the effect of acs-yjcH-actP operon loss on APEC virulence as the previously described with the slightly modified system $[22,46]$. To assess survival/mortality rates were challenged intratracheally with bacteria at $5.5 \times 10^{5} \mathrm{CFU} /$ chick (dose/chick similar to $\mathrm{LD}_{50}$ of wild-type FY26). For systemic infection experiment of chick colibacillosis model, the chick groups were challenged intratracheally with bacteria at $3.0 \times 10^{6} \mathrm{CFU} /$ chick (dose/ chick similar to $\mathrm{LD}_{90}$ of wild-type FY26).

\section{Histological analyses}

The left lungs of the control and APEC-infected ducks were excised, immediately fixed with formalin. The routine histology was performed, and duck lungs were embedded in paraffin. Sections $(4-6 \mu \mathrm{m})$ were cut by rotary microtome and fixed on slides. Sample slides were deparaffinized in xylol, and then rehydrated in ethanol (100\%, 96\%, 80\%, and 70\%). Sections were stained with hematoxylin and eosin (HE). Pathological assessments were performed using a light microscope (Eclipse, Nikon, Tokyo, Japan).

\section{Bacterial cytotoxicity assay}

To investigate the contribution of acetate assimilation system on APEC FY26 cytotoxicity in macrophages, cytotoxicity level was reflected through the lactate dehydrogenase (LDH) release test as previously described [43]. Cells with three replicate wells were infected with each bacteria at a MOI of 5 , and cells culture supernatants after $2 \mathrm{hpi}, 4 \mathrm{hpi}, 8 \mathrm{hpi}$, and $12 \mathrm{hpi}$ were collected and centrifuged to remove any suspended cells. CytoTox 96 nonradioactive cytotoxicity kit (Promega) was used to determine the LDH releases by HD11 cells according to the manufacturer's instructions. The supernatant was incubated with substrate reagent for cytotoxicity kit at room temperature under the dark condition, and the absorbance of the mixture was measured at $490 \mathrm{~nm}$. The spontaneous LDH release was measured by harvesting the supernatant from uninfected cells. Cytotoxicity level was counted as a percentage of the amount of LDH released from infected macrophages relative to that of uninfected cells, which were lysed with $10 \%$ Triton X-100, as follows: (sample LDH release-spontaneous LDH release)/(maximum LDH release-spontaneous LDH release) $\times 100 \%$. Data acquired from at least four individual experiments, and each assay was performed by three biological repetitions.

\section{Results}

The host-induced transcription of APEC actP, yjcH, and acs genes during its infection in macrophages, not happened at non-phagocytic cells

qRT-PCR was used to determine the transcription level of acs-yjcH-actP operon, ackA-pta operon, and satP gene in APEC/ExPEC strains (FY26, CVCC249, and RS218), which were the typical APEC/ExPEC dominant serotypes (O1:K1, O2:K1, and O18:K1) strains and belong to phylogroup B2 and ST95 [22, 27, 47]. qRT-PCR results showed that the transcription levels of $a c s, y j c H, a c t P, a c k A, p t a$, and satP genes in APEC/ExPEC strains (FY26, CVCC249, and RS218) were close those of $d n a E$ (E. coli housekeeping gene) under the routine culture condition at $41{ }^{\circ} \mathrm{C}$ in vitro (Figure 1). When wild-type FY26 was survived in HD11 macrophages, the transcription levels of acs, $y j c H$, and actP were obviously enhanced about 84.3-fold, 81.0-fold, and 78.2-fold at $4 \mathrm{hpi}$, respectively, compared to those under the routine culture condition (long logarithmic phase, LB broth for $6 \mathrm{~h}$ at $\left.41{ }^{\circ} \mathrm{C}\right)(P<0.01)$ (Figure 1A). The transcription levels of $a c s, y j c H$, and $a c t P$ in FY26 were also obviously enhanced at $8 \mathrm{hpi}$, respectively, relative to that for the cultured in vitro $(P<0.01)$ (Figure 1A). Additionally, the similar inducible up-regulation of acs, $y j c H$, and actP transcription could be detected when FY26 infected RAW264.7 at $37{ }^{\circ} \mathrm{C}$ (Figure 1B). However, there were no obvious differences in the transcription of ackA, pta, and satP genes between FY26 invasion in non-phagocytic cells (DF-1 or HEp2) and the cultured at $37^{\circ} \mathrm{C}$ in vitro $(P \geq 0.05)$ (Figure $\left.1 C\right)$. Similarly, during $\mathrm{CVCC} 249$ survival in HD11 macrophages, the transcription levels of $a c s, y j c H$, and actP were obviously enhanced about 68.4-fold, 73.5-fold, and 75.9-fold at $4 \mathrm{hpi}$, respectively, compared to those under the routine culture condition at $41{ }^{\circ} \mathrm{C}(P<0.01)$ (Figure 1D). Similarly, the inducible up-regulation of $a c s, y j c H$, and $a c t P$ transcription could be detected when CVCC249 infected RAW264.7 macrophages at $37^{\circ} \mathrm{C}$, and the transcription levels of $a c s, y j c H$, and $a c t P$ in CVCC249 were enhanced about 70.1-fold, 69.1-fold, and 72.0-fold at $4 \mathrm{hpi}$, respectively, relative to those from in vitro culture $(P<0.01)$ (Figure 1D). Moreover, during RS218 survival in macrophages, the transcription levels of acs, yjcH, and actP were also obviously enhanced when compared with those of the routine culture condition $(P<0.01)$ (Figure $1 \mathrm{E}$ ). However, there was no obvious difference for the transcription of ackA, pta, and satP genes between APEC survival in HD11 macrophages and the in vitro cultured $(P \geq 0.05)$.

These results indicated that the transcription of actP, $y j c H$, and acs genes presented host-induced trait during APEC infection in macrophages, but not in non-phagocytic cells. This characteristic suggested that the acetate 
Figure 1 The transcription levels of $a c s, y j c H, a c t P, a c k A$, pta, and satP genes in APEC/EXPEC strains. QRT-PCR of these genes was determined relative to the transcription level of gene dnaE. Significant differences in GRT-PCR data were identified using one-way ANOVA statistical analysis $\left({ }^{* *} P<0.01\right)$. A The transcriptional differences of $a c s, y j c H$, actP, ackA, pta, and satP genes during FY26 survival in HD11 macrophages relative to that in routine non-infectious condition at $41{ }^{\circ} \mathrm{C}$ in vitro. qRT-PCR data (uninfected FY26 in vitro; infected FY26 at $4 \mathrm{hpi}$ or $8 \mathrm{hpi}$ ) acquired from three individual experiments were used to measure the differences (fold-change) of these genes transcription. B The transcriptional differences of $a c s, y j c H$, actP, ackA, pta, and satP genes during FY26 survival in RAW264.7 macrophages relative to that in routine non-infectious condition at $37^{\circ} \mathrm{C}$ in vitro. QRT-PCR data (uninfected FY26 in vitro; infected FY26 at 4 hpi or 8 hpi) was acquired from three individual experiments. $\mathbf{C}$ The transcription levels of $a c k A$, pta, and satP genes between FY26 invasion in non-phagocytic cells (DF-1 or HEp2) relative to the cultured at $37{ }^{\circ} \mathrm{C}$ in vitro. qRT-PCR data (uninfected FY26 in vitro, infected FY26 in DF-1 cells at 4 hpi, and infected FY26 in HEp2 at $4 \mathrm{hpi}$ ) from three individual experiments were used to measure the differences (fold-change) of these genes transcription. D Transcriptional differences of acs, yjcH, actP, ackA, pta, and satP genes during strain CVCC249 survival in HD11 and RAW264.7 macrophages at $4 \mathrm{hpi}$ relative to the routine condition in vitro. ETranscriptional differences of acs, yjcH, actP, ackA, pta, and satP genes during RS218 survival in HD11 and RAW264.7

macrophages at 4 hpi relative to the routine condition in vitro.

assimilation system encoded by the acs-yjcH-actP operon might play roles in APEC survival and replication within macrophages.

\section{Acetate assimilation system encoded by acs-yjcH-actP operon was essential for APEC growth under acetic acid as the sole carbon source}

To clarify the roles of this acetate assimilation system during APEC infection, the whole acs-yjcH-actP operon was deleted in wild-type FY26, and the single deletion mutants of acs, actP or satP gene were also constructed on strain FY26 (Additional file 1). The complemented strains FY26Cacs-yjcH-actP and FY26CsatP were generated by transformation of plasmids pSTV28-acs-yjcHact $P$ and pSTV28-sat $P$ to the corresponding mutants, respectively. Growth in vitro was first evaluated in a series of analyses. As showed in Figure 2A, the growth curves showed that deletion of acs-yjcH-actP operon or satP gene in strain FY26 had no effect on $E$. coli growth under the LB cultured (rich media) condition. Similarly, there was no difference among the growth curves of wildtype FY26, mutants FY26 $\Delta a c s-y j c H$-actP, FY26 $\Delta a c s$,

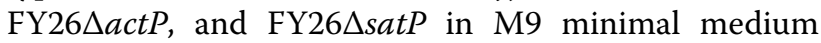
without acetic acid (Figure 2B). For M9 medium plus acetic acid $(60 \mathrm{mM}, 0.36 \%)$, our result showed that satP mutant displayed a slight lag phase compared with the growth curve of wild-type FY26. However, the deletion of acs-yjcH-actP operon in APEC FY26 have a significant alteration in growth curves under M9 medium plus
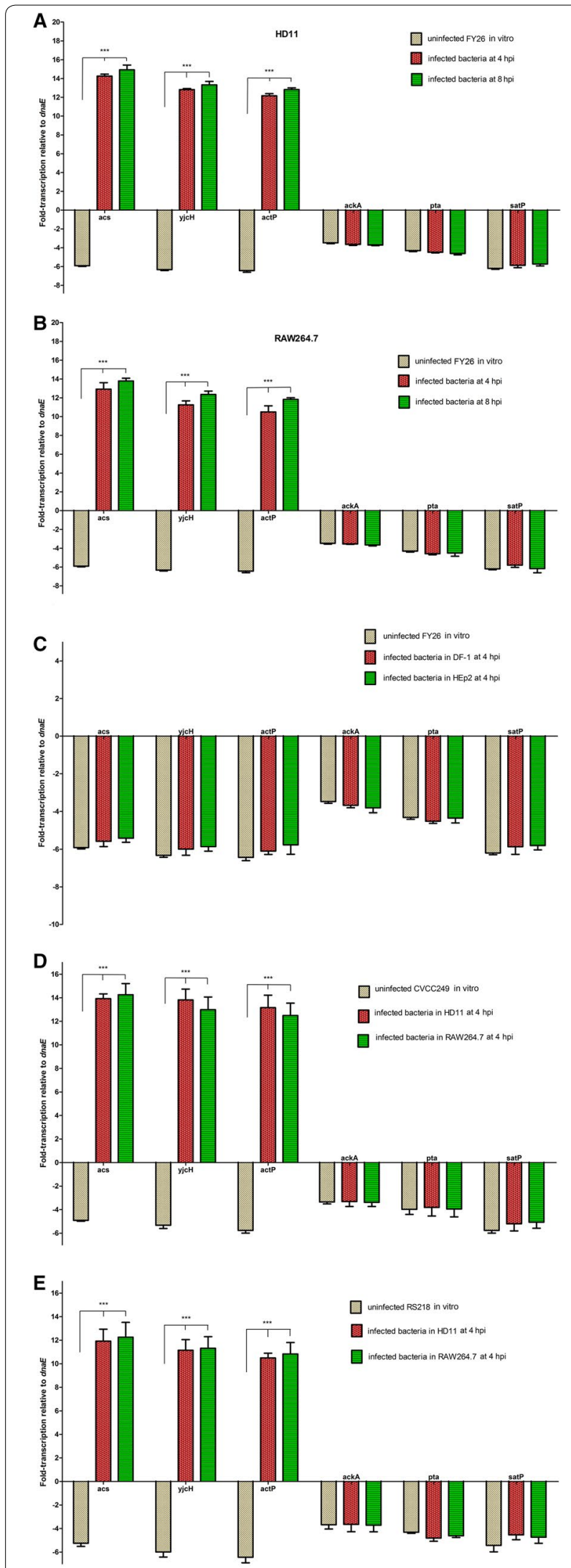
acetic acid condition. This result suggested that acetate assimilation system encoded by acs-yjcH-actP operon was essential for bacterial growth when acetic acid acted as the sole carbon and energy source (Figure 2C). FY26 $\Delta a c s$ and FY26 $\Delta a c s-y j c H$-actP grew equally in minimal medium (M9 plus $60 \mathrm{mM}$ acetic acid) and display a longer lag phase relative to the growth curve of mutant FY26 $\triangle a c t P$. Moreover, the growth of the complemented strains FY26Cacs-yjcH-actP and FY26CsatP was restored to the level of wild-type FY26 (Figure 2C).

\section{Acetate assimilation system encoded by acs-yjcH-actP operon was essential for APEC intracellular replication within macrophages}

Previous studies have revealed that APEC can survive and replicate within macrophages [13, 40, 48]. However, the metabolic strategies utilized by intracellular APEC to enhance proliferative capacity in host phagocytosis cells remain poorly understood. Acetate can act as a carbon source to support energy homeostasis in prokaryotic or eukaryotic cells. As expected, the replication ability of the mutants (lacking acs, actP, or the whole operon) survival in HD11 macrophages was significantly impaired relative to that of wild-type FY26. As shown in Figure 3A, the survival rate of the FY26 2 acs at 4 hpi and 8 hpi were decreased to $72.5 \%$ and $46.2 \%$ relative to those of wild-type FY26, respectively $(P<0.01)$. Similarly, the survival rate of the FY26 $\triangle a c t P$ at $4 \mathrm{hpi}$ and 8 hpi were just only about $74.1 \%$ and $44.9 \%$ compared to that of wild-type FY26, respectively $(P<0.01)$. Additionally, consistent with the single deletion of acs or actP gene in FY26, the results showed that deletion of acs$y j c H$-actP operon impaired the APEC replication within HD11 macrophages $(P<0.01)$. The survival of the complemented strain FY26Cacs-yjcH-actP was restored to the level of wild-type FY26. However, there was no obvious difference between FY26 $\Delta$ satP and wild-type FY26 $(P \geq 0.05)$. As shown in Figure 3B, the replication ability of these mutants (lacking acs, $a c t P$, or the whole operon) survival in RAW264.7 macrophages was also significantly impaired compared with that of wild-type FY26 $(P<0.01)$.

Immunofluorescence assay was performed to determine whether acs-yjcH-actP operon loss affected the APEC survival or replication within HD11 macrophages. At a MOI of 5, the presence of bacteria can be detected in more than $96 \%$ of infected cells at the initial time point (2 hpi). Numbers of bacteria per infected HD11 cells at different time points were divided into the following categories: $\leq 2,3$ to 5,6 to 10 , and $\geq 11$. The number of bacteria (FY26, FY26 $\Delta a c s-y j c H$-actP, and FY26Cacs-yjcH-actP) in 100 infected cells at 2 hpi and 4 hpi was counted. As a result, intracellular bacteria

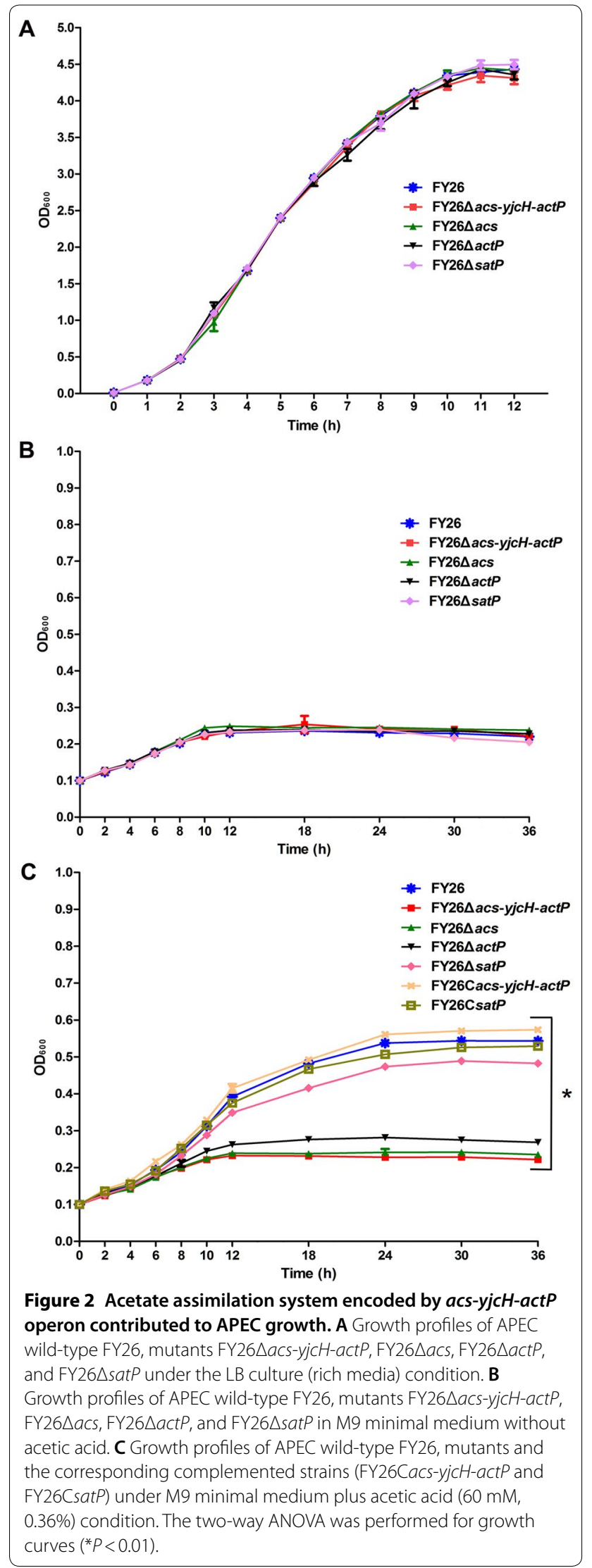

B

Figure 2 Acetate assimilation system encoded by acs-yjcH-actP operon contributed to APEC growth. A Growth profiles of APEC and FY26 $\triangle$ satP under the LB culture (rich media) condition. B Growth profiles of APEC wild-type FY26, mutants FY26 $\triangle$ acs-yjcH-actP, the corresponding complemented strains (FY26Cacs-yjcH-actP and FY26CsatP) under M9 minimal medium plus acetic acid $(60 \mathrm{mM}$ curves $\left({ }^{*} P<0.01\right)$ 

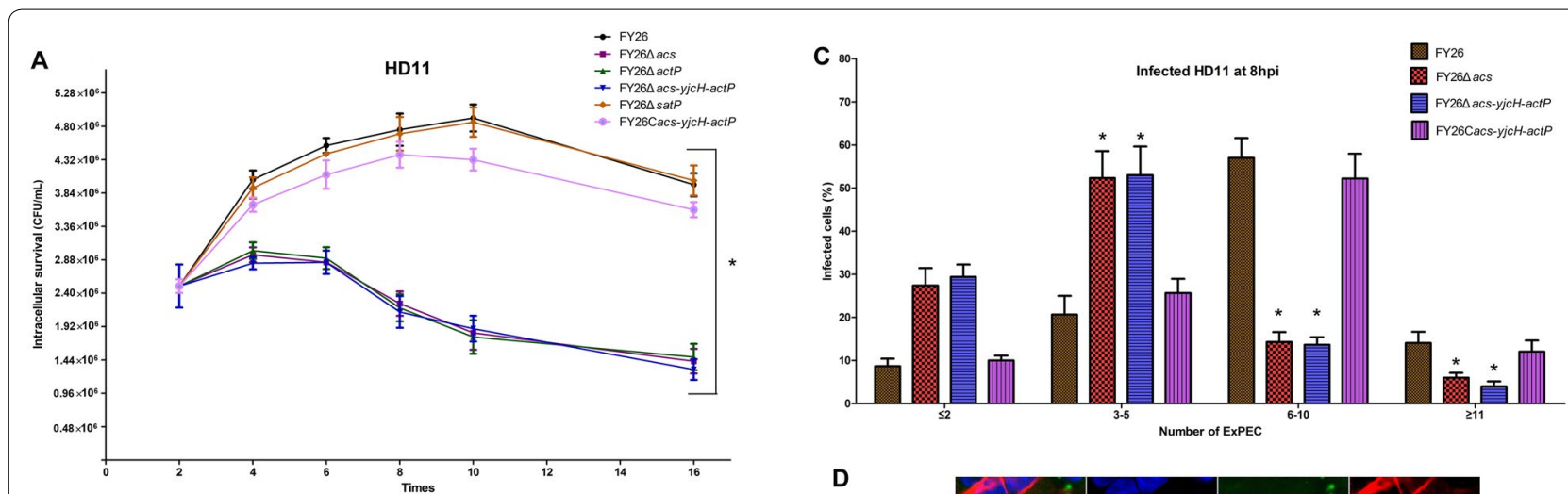

D
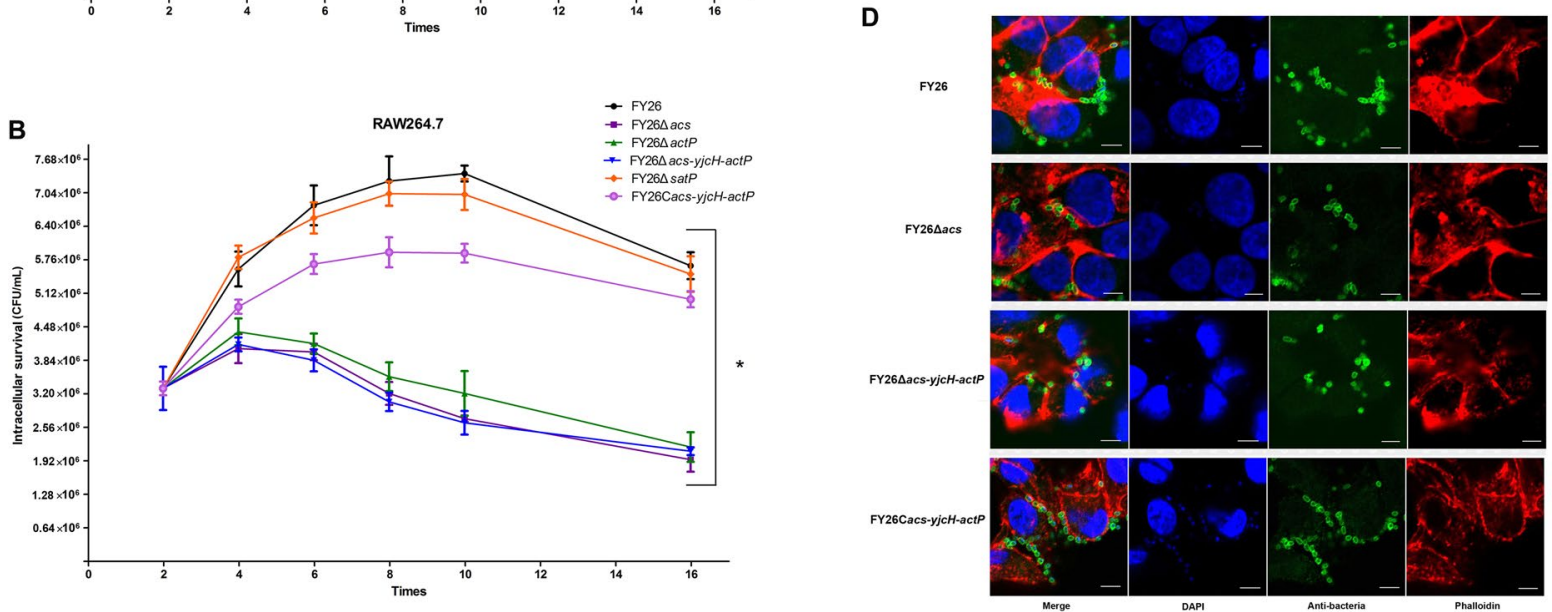

Figure 3 Acetate assimilation system encoded by acs-yjcH-actP operon contributed to APEC intracellular replication within macrophages. A Deletion of acs-yjcH-actP operon impaired FY26 intracellular replication within HD11 macrophages. The survival rates of wild-type

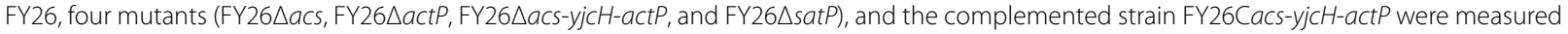
to investigate the contribution of the acetate assimilation system on APEC proliferation. B To determine the contribution of acetate assimilation system on APEC replication within RAW264.7 macrophages. Macrophages were infected at a MOI of 10. Data acquired from at least four individual experiments, and each assay was performed by three biological repetitions. The significant differences were identified using two-way ANOVA analysis $\left({ }^{*} P<0.01\right)$, and the mean values \pm SEs were shown in each plotting. C, D Determine the effect of acetate assimilation system on APEC replication within macrophages by immunofluorescence assays. HD11 cells were infected at a MOI of 5. C Quantification of wild-type FY26, FY26 $\triangle a c s$, FY26 $\triangle a c s-y j c H-a c t P$, and FY26Cacs-yjcH-actP intracellular proliferation in macrophages was indicated. The percentage of infected HD11 cells containing $\leq 2,3$ to 5,6 to 10 , and $\geq 11$ bacteria per cell at 8 hpi was counted by fluorescence microscopy. Data for quantification of bacteria proliferation was acquired from more than 100 infected cells for three independent experiments. The significant differences were identified using one-way ANOVA analysis $\left({ }^{*} P<0.01\right)$, and the mean values \pm SEs were shown. D Representative confocal microscopy images of wild-type FY26, FY26 $\triangle a c s$, FY26 $\triangle a c s-y j c H-a c t P$, and FY26Cacs-yjcH-actP intracellular proliferation in macrophages at 8 hpi were shown. The infected HD11 cells were fixed and labeled with DAPI and Phalloidin (actin staining;TRITC conjugated). Bacteria were incubated with an anti-APEC antibody and immediately labeled with a fluorescent secondary antibody (Alexa 488, green). Scale bar $=10 \mu \mathrm{m}$.

were generally less than 5 , and the statistical differences were not observed by immunofluorescence at early time points. As shown in Figure 3C, the majority ( 71.1\%) of the HD11 macrophages infected with wild-type FY26 contained $\geq 6$ bacteria at $8 \mathrm{hpi}$, and the percentage of infected cells containing 6 to 10 bacteria was $\sim 57 \%$. In contrast, the percentage of FY26 $\triangle a c s-y j c H$-actP infected macrophages containing $\geq 6$ bacteria decreased significantly to approximately $\sim 20.3 \%$ at $8 \mathrm{hpi}$. Bacteria counting of FY26 $\triangle a c s$ infected macrophages was similar to the result of FY26 $\Delta a c s-y j c H$-actP (Figure 3C). Furthermore, bacteria counting revealed that the replication ability of the complemented FY26Cacs-yjcH-actP was restored to the similar level of wild-type FY26. Representative fluorescence microscopy images of the strains replication within HD11 macrophages at 8 hpi were shown in Figure 3D. These results clarified that acetate assimilation system encoded by acs-yjcH-actP operon acted as an intracellular survival factor, and the acetate metabolic requirement of APEC promoted its intracellular proliferation within macrophages. 


\section{The acetate assimilation system was required for APEC inducing macrophage injury}

Besides being a fuel for central metabolism, the emerging studies indicate the functional role of the acetate metabolism in host central energy homeostasis and critical correlation with multiple host physiological features $[17,49]$. In independent experiments, we assessed the effect of acetate assimilation system on host cell damage during APEC interaction with macrophages. The cytotoxic effect of acs, actP or satP mutants to macrophages (HD11 and RAW264.7) was determined by quantifying the amount of the lactate dehydrogenase (LDH) released by the infected macrophages. LDH released from infected HD11 macrophages was detected at four time points ( $2 \mathrm{hpi}, 4 \mathrm{hpi}, 8 \mathrm{hpi}$, and $12 \mathrm{hpi}$ ). The data showed that the amount of LDH release was gradually increased during FY26 interaction with HD11 cells, whereas macrophages exposed to the acs, actP, or acs-yjcH-actP mutant exhibited decreased LDH release level relative to that of wildtype FY26 $(P<0.01)$ (Figure $4 \mathrm{~A})$. Furthermore, the LDH testing results revealed that the cytotoxicity level exposed to complemented FY26Cacs-yjcH-actP was restored to the similar level of wild-type FY26. The deletion of satP gene in FY26 had no obvious effect on cytotoxicity in HD11 macrophages $(P>0.05)$. Moreover, the LDH testing results demonstrated that the cytotoxic levels during RAW264.7 macrophages exposed to the acs, actP, and acs-yjcH-actP mutants were significantly lower relative to that of wild-type FY26 $(P<0.01)$ (Figure 4B). Similarly, the cytotoxicity on RAW264.7 exposed to the complemented FY26Cacs-yjcH-actP was restored to the similar level of the wild-type FY26 $(P>0.05)$. The deletion of satP gene in FY26 had no obvious effects on cytotoxicity to RAW264.7 macrophages $(P>0.05)$. Mitochondrion toxicity was also conducted in this study, but there were no differences on mitochondrion toxicity for macrophages exposed to between wild-type FY26 and mutants (data not shown). Taken together, these data indicated that the acetate assimilation system was required for APEC inducing macrophage injury during its intracellular replication.

\section{Over-expression of APEC acetate assimilation system enhanced the production of nitric oxide (NO) and proinflammatory cytokines in infected macrophages}

Once infected by a pathogen, macrophages can secrete large amounts of pro-inflammatory and anti-inflammatory cytokines and interleukins, and enhance the production of nitric oxide (NO). An important incentive for bacteria successful survival and proliferation within infected macrophages is the excessive inflammation due to the imbalance of pro-inflammatory and anti-inflammatory responses, accompanied by immunity failure and body inflammatory damage [4]. To investigate whether the over-expression of acetate assimilation system could induce inflammatory responses of APEC infected macrophages, we determined the cytokine responses between wild-type FY26 and FY26 $\triangle a c s-y j c H$-actP infected HD11 macrophages at 4 hpi by qRT-PCR. Referring to recent research $[15,37,39,50]$, the gene transcription involved in pro-inflammatory interleukins (IL-1 $\beta$, IL-6, IL-8, IL-12 $\alpha$, and IL-12 $\beta$ ), tumor necrosis factor- $\alpha$ (TNF- $\alpha$ ), inducible nitric oxide synthase (iNOS), and anti-inflammatory cytokines (IL-4, IL-10, and IL-13) was examined. As shown in Figure 5A, the transcriptional levels of these targeted genes encoding pro-inflammatory cytokines (IL-1 $\beta$, IL-6, IL-8, IL-12 $\beta$, and TNF- $\alpha$ ) and iNOS were significantly up-regulated in FY26 infected HD11 at $4 \mathrm{hpi}$ and $8 \mathrm{hpi}$ relative to that of the control (uninfected HD11 cultures), especially the excessive expression of IL-8, TNF, and iNOS $(P<0.01)$. Unlike the pro-inflammatory cytokines, the transcription of anti-inflammatory cytokines (IL-4, IL-10, and IL-13) presented a lower level in infected HD11, which were similar to that of the uninfected HD11 cultures $(P>0.05)$ (Figure 5A). This result indicated that infected HD11 macrophages presented excessively pro-inflammatory responses. However, deletion of acs-yjcH-actP genes in FY26 had obvious effects on the transcription of the pro-inflammatory cytokines in infected HD11 macrophages. qRT-PCR results showed that the transcription of IL- $1 \beta$, IL-6, IL-8, IL- $12 \beta$, TNF- $\alpha$, and iNOS was obviously down-regulated in FY26 $\triangle a c s-$ $y j c H$-actP infected macrophages compared to that in wild-type FY26 infected cultures at 4 hpi $(P<0.01)$ (Figure $5 \mathrm{~B})$. Similarly, the transcription of the pro-inflammatory cytokines in FY26Cacs-yjcH-actP infected macrophages was restored to the similar level for the wild-type FY26 infected condition $(P>0.05)$.

To determine the effect of acs-yjcH-actP deletion on proinflammatory responses in RAW264.7 macrophages, ELISA assay was conducted to evaluate the production of nitric oxide (NO) and proinflammatory cytokines (IL$1 \beta$, IL-6, IL-8, IL-12 $\beta$, and TNF- $\alpha$ ) in RAW264.7 macrophages. As shown in Figure $5 \mathrm{C}$, the release levels of NO, IL-1 $\beta$, IL-6, IL-8, IL-12 $\beta$, and TNF- $\alpha$ for FY26 $\Delta a c s-$ $y j \mathrm{cH}$-actP infected RAW264.7 macrophages were significantly lower than that of wild-type FY26 infected cultures at $8 \mathrm{hpi}(P<0.01)$. The release levels of NO, IL-1 $\beta, \mathrm{IL}-6$, IL-8, IL-12 $\beta$, and TNF- $\alpha$ in FY26Cacs-yjcH-actP infected RAW264.7 macrophages were partially restored to the levels for the wild-type FY26 infected cell cultures $(P>0.05)$. Moreover, the cytokine production at a later time point (16 hpi) was measured. As shown in Figure $5 \mathrm{D}$, the release levels of NO, IL-1 $\beta$, IL-6, IL-8, IL-12 $\beta$, and TNF- $\alpha$ for FY26 $\Delta a c s-y j c H$-actP infected RAW264.7 macrophages were also decreased relative to that of wild-type FY26 infected cultures at 16 hpi $(P<0.01)$. In 

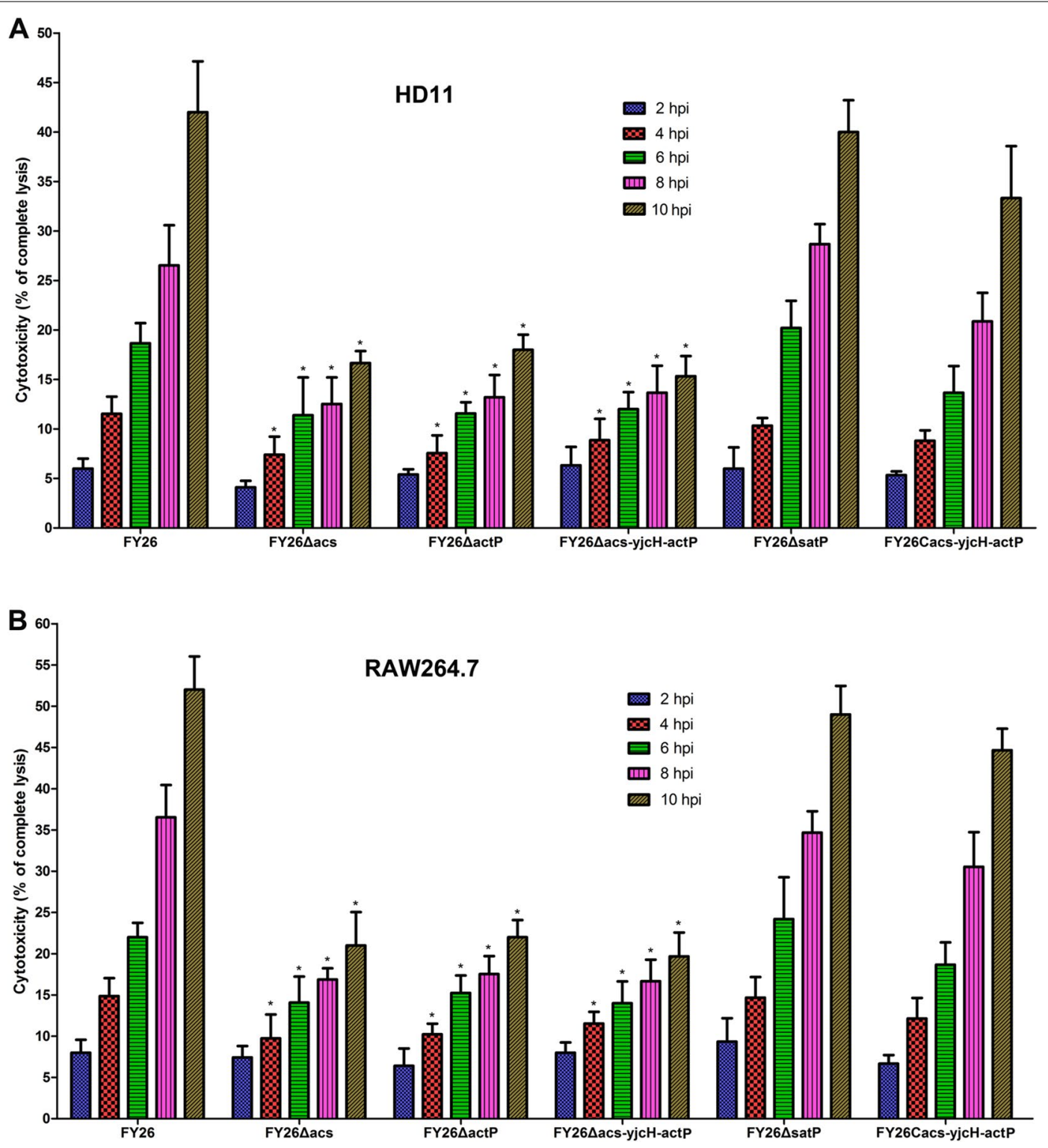

Figure 4 Acetate assimilation system encoded by acs-yjcH-actP operon was required for APEC-induced cytotoxicity to macrophages. A To investigate the cytotoxic effect of acs, actP or satP mutants on HD11 macrophages. Cells culture supernatant after 2 hpi, 4 hpi, 8 hpi, and 12 hpi was collected to determine the LDH releases by HD11 cells. Cytotoxicity level was counted as a percentage of the amount of LDH released from infected macrophages relative to that of uninfected cells lysed with $10 \%$ Triton X-100. B To determine the cytotoxic effect of acs, actP or satP mutants on RAW264.7 macrophages. Data acquired from at least four independent experiments performed in triplicate, and the mean values \pm the standard errors were shown. Statistical differences were determined using two-way ANOVA analysis $\left({ }^{*} P<0.01\right)$.

this section, the results showed that the over-expression of APEC acetate assimilation system promoted excessively pro-inflammatory responses through enhancing the production of nitric oxide (NO) and proinflammatory cytokines in infected macrophages. The recent research shows that the acetate plays a critical role in the modulation of host inflammatory response [17, 49, 51]. Therefore, the host acetate consumption during APEC survival in macrophages might cause host immunomodulatory disorders and excessively pro-inflammatory responses.

\section{The acetate assimilation system was increasingly expressed during APEC early colonization in lung tissues in vivo for duck model}

To further determine whether the acetate assimilation system encoded by acs-yjcH-actP operon affected the 


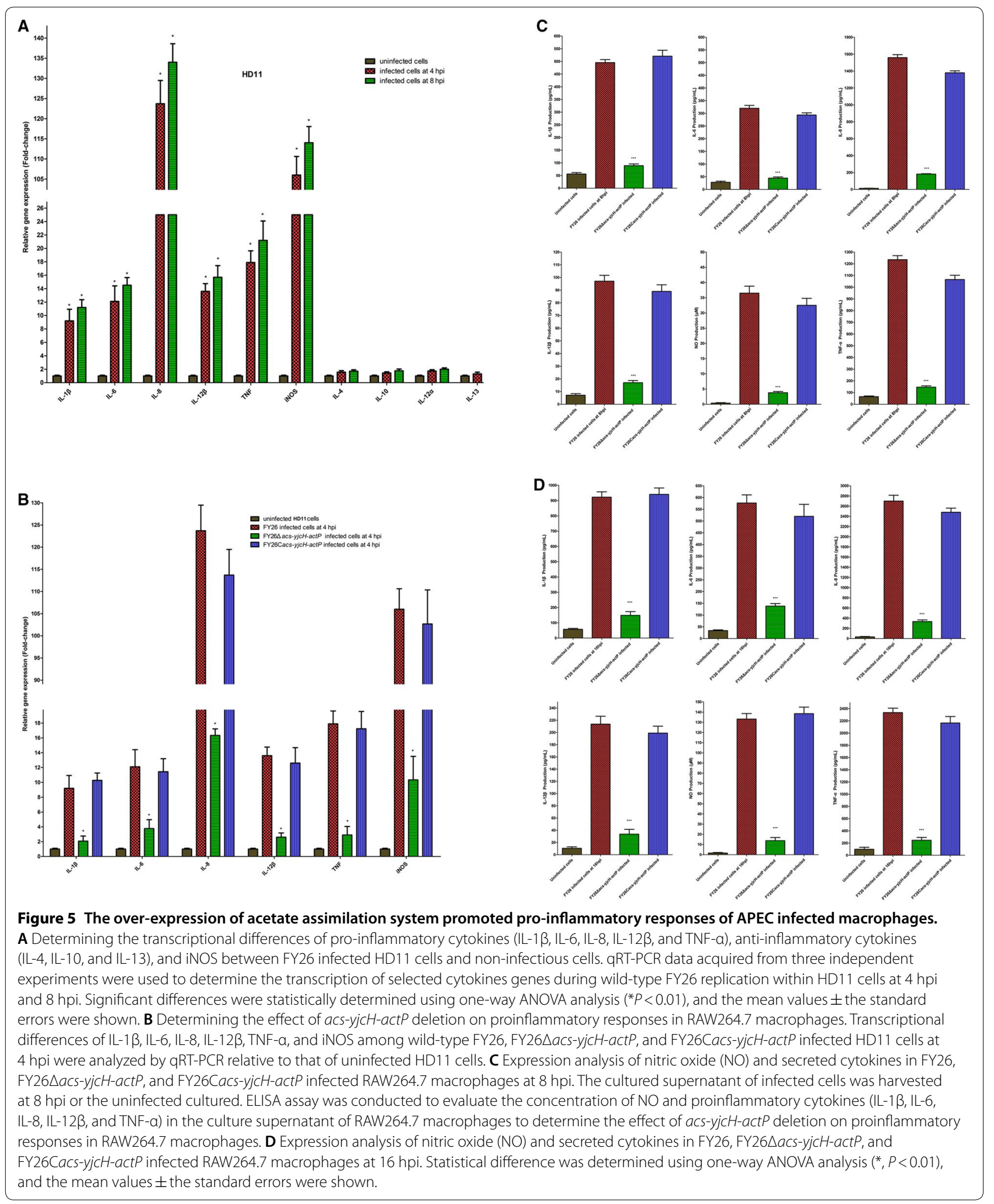


virulence of wild-type FY26, we detected the expression of acetate assimilation system during FY26 colonization in vivo in avian colisepticemia model. The duck is used as a typical avian infection model to evaluate the virulence of APEC isolates. The 7-day-old ducklings were inoculated intratracheally with bacteria at $2.0 \times 10^{6} \mathrm{CFU}$, and qRT-PCR was used to determine the transcription of $a c s, y j c H$, actP, and satP genes during FY26 colonization in duck organs (lung, liver and spleen) and proliferation in blood in vivo at $24 \mathrm{hpi}$. As shown in Figure 6A, our result indicated that the transcription levels of acs, $y j c H$, and $a c t P$ were up-regulated by 68.4-fold, 66.3-fold, and 64.3-fold during FY26 colonization in duck lung tissues compared with that from the routine culture in vitro $(P<0.01)$ (Figure 6A). However, the transcription levels of $a c s, y j c H$, actP, and satP genes for wild-type FY26 isolated from bacteremia in vivo were close to that from the routine culture in vitro $(P>0.05)$ (Figure 6A). Moreover, the transcription levels of these genes during wild-type FY26 colonization in duck livers or spleen were also close to that of $d n a E$, and didn't present induced up-regulation (data not shown). Similar to the results of duck infection model, the transcription levels of $a c s, y j c H$, and $a c t P$ were obviously up-regulated during FY26 colonization in chick lung tissues compared with those from the routine culture $(P<0.01)$ (Figure 6B).

\section{Deletion of actP/yjcH/acs genes attenuated APEC virulence and colonization capability in avian lungs in vivo for avian infection models}

To investigate the effect of acs-yjcH-actP deletion on the virulence of APEC strain FY26, the mortality rates of wild-type FY26, FY26 $\Delta a c s$, FY26 $2 a c s-y j c H$-actP, and the complemented FY26Cacs-yjcH-actP was determined in the duck model. Each duckling was challenged intratracheally with bacteria at $5.0 \times 10^{5} \mathrm{CFU}$. As shown in Figure 7A, the survival curves indicated that FY26 $\triangle a c s$ and FY26 $\triangle a c s-y j c H$-actP decreased the mortality rates and postponed the death peak of the infected ducks compared with that of wild-type FY26 $(P<0.01)$. Moreover, the mortality rate of the complemented FY26Cacs-yjcHactP was restored to the similar level of wild-type FY26 $(P>0.05)$. However, the acs-yjcH-actP operon deletion had no effect on the APEC virulence in the murine sepsis model (data not shown). The result indicated that the acetate assimilation system conferred a fitness advantage of APEC infection in duck model relative to that of the murine sepsis model.

To measure the effect of acs-yjcH-actP operon deletion on APEC colonization in vivo, the systemic infection experiment of the duck model was performed to assess the bacteria proliferation in duckling organs and septicemia level. As shown in Figure 7B, the early colonization capacity in duckling lungs among the four strains was determined at $24 \mathrm{hpi}$. The mutants FY26 $\Delta a c s$ and FY26 $\triangle a c s-y j c H$-actP exhibited decreased colonization capacity in duckling lungs relative to that of wildtype FY26 and the complemented FY26Cacs-yjcH-actP $(P<0.01)$. About $65 \%$ of the ducks infected with the

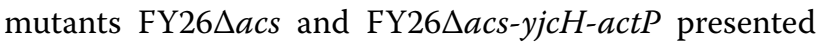
bacteremia symptoms compared to the ducks challenged by wild-type FY26, suggesting that the loss of acs-yjcH-actP operon might impair APEC capability to pass through the immune defense of duck lung tissues into the bloodstream $(P<0.01)$. However, the septicemia for the alive ducklings infected with the mutants was not detected. For the ducklings presenting bacteremia symptoms, the statistics of bacteremia levels showed that bacteria proliferation in the blood for FY26 $\triangle a c s$ and FY26 $\triangle a c s-y j c H$-actP infected ducks had no significant difference with that of wild-type FY26 and the complemented FY26Cacs-yjcH-actP (Figure 7B) $(P>0.05)$, suggesting acetate assimilation system encoded by acsyjcH-actP operon was not involved in bacteria proliferation in blood in vivo. Moreover, there was no significant difference for the bacteria proliferation/colonization of these strains among duckling spleen, liver, brain, and kidney tissues (data not shown). The results of systemic infection experiment illustrated that the acetate assimilation system conferred an advantage of the APEC early colonization in duck respiratory system in vivo.

Similar to the results of duck infection model, deletion of $a c t P / y j c H / a c s$ genes attenuated APEC virulence and colonization capability in avian lungs in vivo for chicken infection model. As shown in Figure $7 \mathrm{C}$, the survival curves indicated that FY26 $\Delta a c s-y j c H$-actP decreased the mortality rate and postponed the death peak of the infected chicks compared with that of wildtype FY26 $(P<0.01)$. The mutant FY26 $\Delta a c s-y j c H$-actP exhibited decreased colonization capacity in chick lungs relative to that of wild-type FY26 and the complemented FY26Cacs-yjcH-actP $(P<0.01)$ (Figure 7D). Similar to the duck model, $60 \%$ of the chicks infected with the mutant FY26 $\Delta a c s-y j c H$-actP presented bacteremia symptoms compared to the ducks challenged by wild-type FY26. For the chicks presenting bacteremia symptoms, the statistics of bacteremia levels showed that bacteria proliferation in the blood for FY26 $\triangle a c s$ and FY26 $\triangle a c s-y j c H$-actP infected ducks had no significant difference with that of wild-type FY26 and the complemented FY26Cacs-yjcH-actP (Figure 7D).

We conducted histopathological analysis of the duck lungs at $12 \mathrm{hpi}, 24 \mathrm{hpi}, 36 \mathrm{hpi}$, and $48 \mathrm{hpi}$ after duckling challenged intratracheally with four strains (wild-type FY26, the mutants FY26 $\Delta a c s$, FY26 $2 a c s-y j c H$-actP, and the complemented FY26Cacs-yjcH-actP), and PBS 

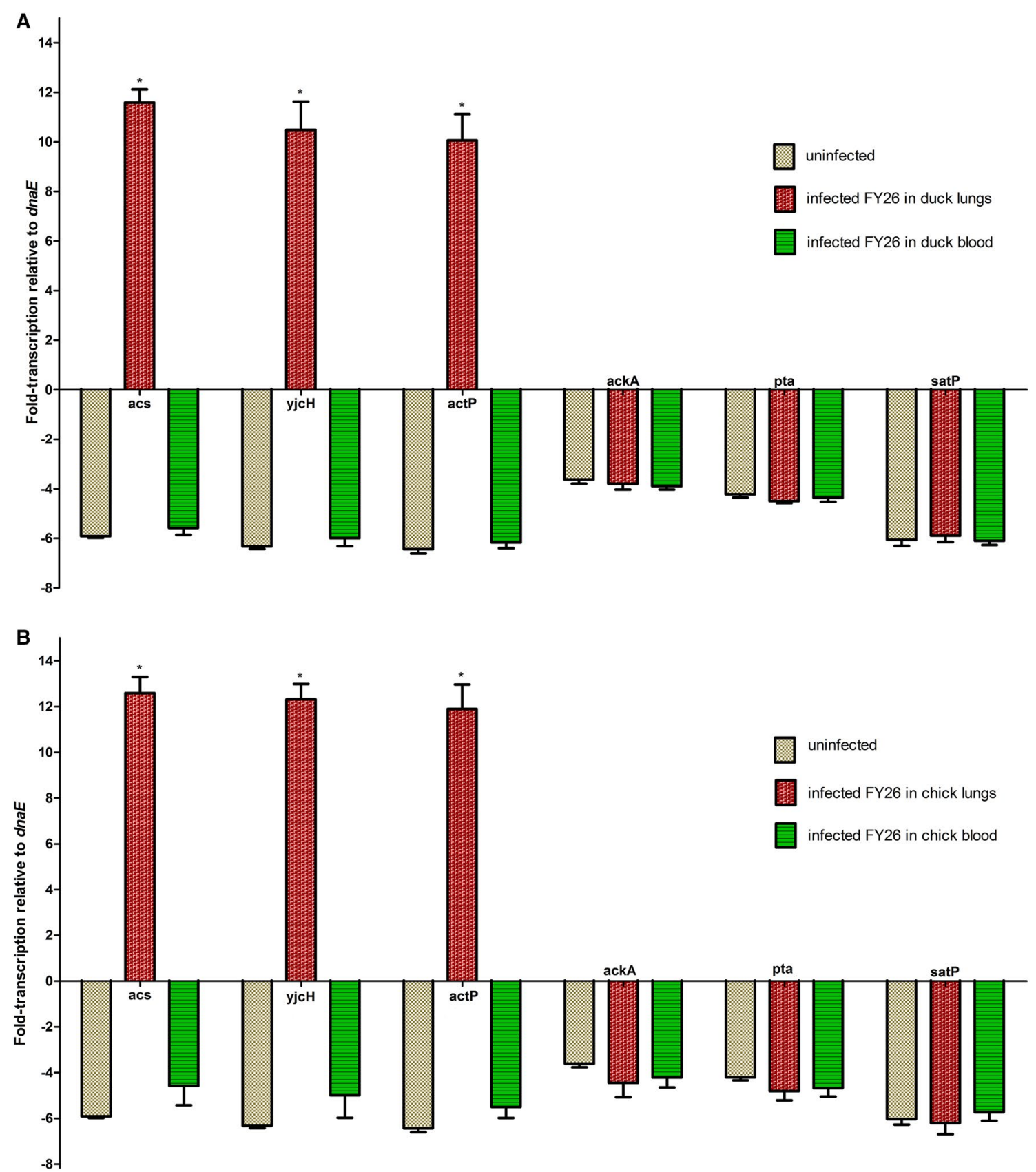

Figure 6 The expression of acetate assimilation system encoded by acs-yjcH-actP operon was highly induced during APEC early colonization in lung tissues in vivo. A For duck model, qRT-PCR data acquired from three individual experiments were used to determine the transcription of $a c s, y j c H$, actP, satP, ackA, and pta genes during wild-type FY26 colonization in duck organs in vivo at 24 hpi. Transcriptional differences of these genes were determined relative to that of the routine condition in vitro. B For chick model, transcriptional differences of acs, yjcH, actP, satP, ackA, and pta genes during FY26 colonization in chick tissues in vivo at 24 hpi were determined relative to that of the routine condition in vitro. Significant differences of qRT-PCR results were identified using one-way ANOVA statistical analysis $\left({ }^{*} P<0.01\right)$.

buffer was used as the negative control. As shown in Figure $7 \mathrm{E}$, panels $\mathrm{a}-\mathrm{d}$, the peribronchial structure of the lung sections for PBS-inoculated ducks was intact at each time point. The parabronchial lumens and pulmonary alveolus were open and aerated, and almost none inflammatory cells infiltrated around the lumen of PBS-inoculated tissue sections. In contrast to the lungs from the uninfected ducks, we observed 

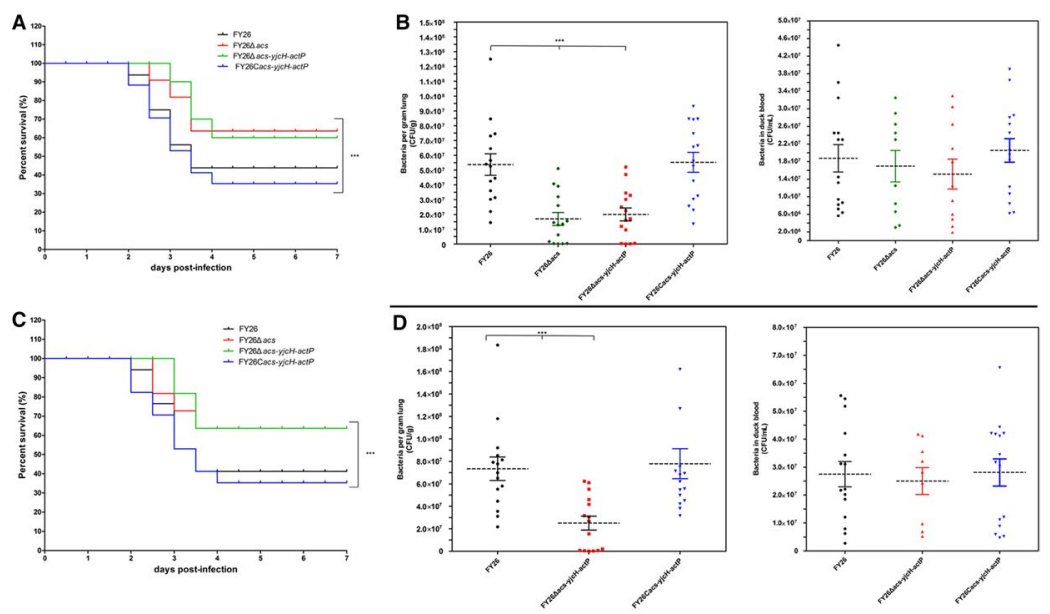

\section{E}

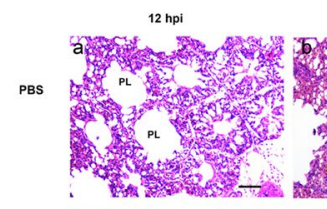

$24 \mathrm{hpi}$

$36 \mathrm{hpi}$

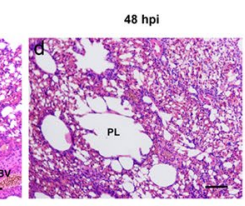

FY26
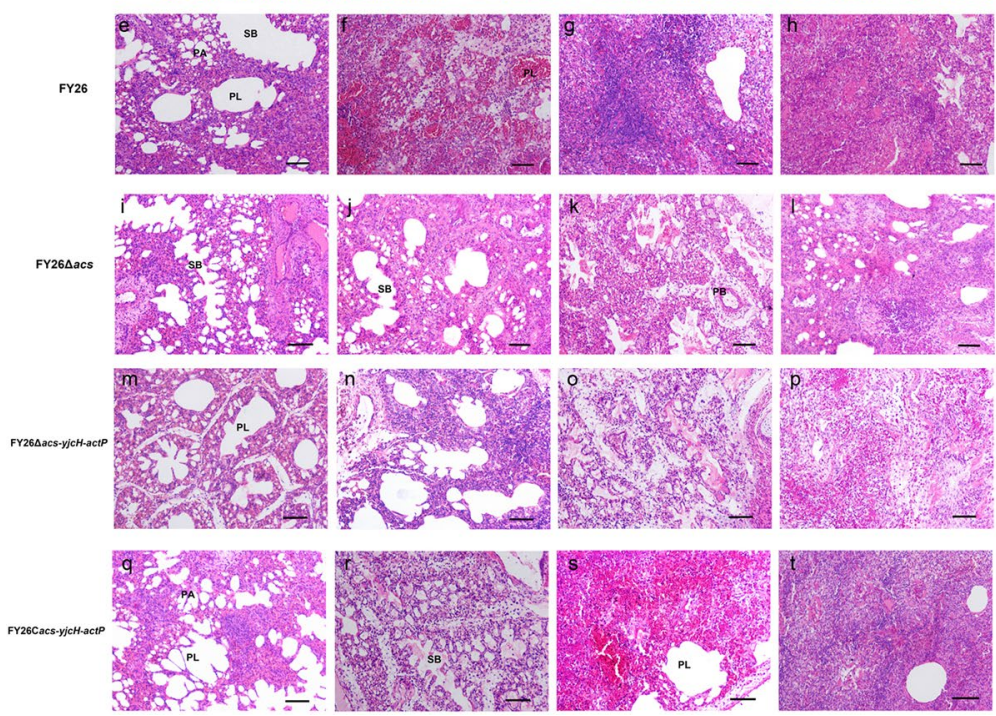

Figure 7 The acetate assimilation system encoded by acs-yjcH-actP operon contributed to APEC virulence and phenotype fitness. A The mortality rates of wild-type FY26 and variants were determined by duck model to investigate the effect of acs-yjcH-actP deletion on the virulence of APEC. Survival rate was measured after 7 days post-infection. The two-way ANOVA was performed for survival assays $\left({ }^{*} P<0.01\right)$. B Determining the effect of acs-yjcH-actP operon deletion on APEC colonization in vivo. Systemic infection experiment of the duck infection model was performed to assess the bacteria proliferation in duckling organs and blood at $24 \mathrm{hpi}$. Statistical significances were identified using a nonparametric MannWhitney $U$ test $\left({ }^{*} P<0.01\right)$. C The mortality rates of wild-type FY26, the mutant FY26 $\triangle$ acs-yjcH-act $P$, and the complemented FY26Cacs-yjcH-actP were determined by chick model. D Systemic infection experiment of the duck infection model was performed to assess the bacteria proliferation in chick organs and blood at 24 hpi. E Histopathology of lungs from ducks infected intratracheally with wild-type FY26, FY26 $\triangle a c s, F Y 26 \triangle a c s-y j c H$-actP, FY26Cacs-yjcH-actP, and the negative control PBS. Duck lungs at 12 hpi, 24 hpi, 36 hpi, or 48 hpi were fixed, and sections were stained with HE. The pathological changes of the lungs were observed under a light microscope. a- $d$ The image observation of lung sections from PBS-inoculated ducks at 12 hpi, 24 hpi, 36 hpi, or 48 hpi, respectively. e-h The lesions of lung sections from FY26-infected ducks at four time points. i-I The lesions of lung

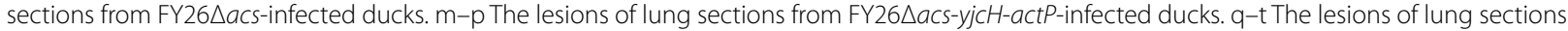
from FY26Cacs-yjcH-actP-infected ducks. Scale bars $100 \mu \mathrm{m}$. BV, blood vessel; PB, primary bronchus; SB, secondary bronchus; PL, parabronchial lumen; PA, pulmonary alveolus. 
the severe pathological changes in the lungs from the FY26-infected ducks (Figure 7E, panels e-h). In the lungs of FY26-challenged duckling ( $24 \mathrm{hpi})$, the interval between the pulmonary alveolus was significantly widened, and a large number of lymphocytes infiltrated in the interval. The other lesions contained the exudation in the bronchioles, alveolar congestion, and capillary interstitial congestion in the pulmonary interstitium (Figure 7E, panel f). With the persistence of APEC infection in duck lungs, the highly inflamed and congested areas were observed in the infected lungs at $36 \mathrm{hpi}$ or $48 \mathrm{hpi}$, accompanied with obvious consolidation of lung tissues (Figure 7E, panels g-h). Deletion of acs-yjcH-actP genes decreased the lesions progress relative to that of FY26-infected ducks (Figure 7E). The relatively light pathological changes and less severe inflammation were present in the lungs

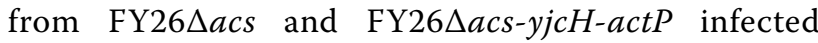
ducks (Figure 7E). Infection with the complemented FY26Cacs-yjcH-actP caused severe inflammation and pathological changes of the lung at $24 \mathrm{hpi}$, similar to that caused by wild-type FY26.

These results illustrated that the acetate assimilation system encoded by acs-yjcH-actP operon not only acted as an APEC intracellular survival factor but also a virulence factor during avian infection route. The acetate assimilation system might facilitate APEC to escape from phagocytes clearance of respiratory system and promote its to enter the bloodstream to cause sepsis and multisystemic infection.

\section{Discussion}

APEC/ExPEC holds extraintestinal infection-specific virulence properties, including many known adhesins, invasins, serum resistance factors, iron acquisition systems, typical transcriptional regulators, and etc., which are clearly distinguished from virulence features of IPEC [22, 25, 26]. When APEC/ExPEC infect specific extraintestinal niches, it must evade or escape host immune defense. During ExPEC colonizing human urinary tract, avian respiratory system, and central nervous system, macrophages play critical roles in host defenses to suppress ExPEC infection [4, 52]. However, macrophages fail to clear out highly virulent ExPEC, especially K1 $E$. coli, and ExPEC can spread in the bloodstream to cause septicemia and fatal multisystemic infection [13, 41]. Nowadays, several authors have proposed that APEC/ ExPEC is a facultative intracellular pathogens, and more and more evidence reveal that intracellular persistence and replication within macrophages is essential for APEC extraintestinal dissemination [13, 30, 40, 41, 53]. It is well-known that intracellular bacterial life cycle mainly consists of three stages: endocytosis and invasion; persistence and proliferation in the cytosol or PCVs of infected host cells; evasion and recolonization of new host cells. APEC requires intracellular survival factors to maintain the intracellular life cycle during its infection in macrophages. For example, K1 capsule is an important survival factor to promote APEC inhibiting the immune response modulation and resistance to phagolysosomes killing $[13,54]$. Until now, there is still no systematic interpretation of APEC intracellular survival/proliferation mechanism, and intracellular survival factors that were especially involved in pathometabolism need to be further revealed.

In this study, we identified that the acs-yjcH-actP operon encoding acetate assimilation system in typical APEC/ExPEC dominant serotypes (O1:K1, O2:K1, and O18:K1) strains presented the host-induced transcription during its infection in macrophages (HD11 and RAW264.7). The phenomenon indicated that this acetate assimilation was activated during APEC infection in macrophages and presented the dominant state relative to acetate dissimilation. More importantly, acetate can be used as the sole carbon source of $E$. coli and other microbiota [17-20]. It seemed that is an inseparable coordination between APEC intracellular survival and expression of acetate assimilation system. The roles of this acetate assimilation system in APEC replication within macrophages were further determined. Recent evidences reveal that the intracellular pathogen can exploit its metabolic pathways to uptake host nutrient sources, aiming to enhance the active survival and proliferation in host intracellular compartments $[4,6,7,55]$. Lactate dehydrogenases (LDHs) is an important survival factor of Neisseria gonorrhoeae to uptake host-derived lactate, which is the critical carbon source for survival/replication of N. gonorrhoeae in phagocytic or epithelial cells [56]. Shigella can reprogram the central metabolism of infected host cells to produce redundant pyruvate, which acts as a favorable energy source to maintain Shigella intracellular growth [2]. The metabolic requirements of APEC proliferation in biofilm-like intracellular bacterial communities (IBCs) during its urinary tract infection, and galactoside metabolism promote UPEC colonization in bladder epithelial cells [5]. Our result showed that acetate assimilation system encoded by acs-yjcH-actP operon acted as a novel intracellular survival factor to promote APEC replication within macrophages. Our research might be the first to unravel the metabolic crosstalk of APEC intracellular survival or replication within macrophages, and acetate metabolic requirement might be an important strategy of APEC/ExPEC pathometabolism. However, the host-induced expression of acetate assimilation system didn't happen when APEC invasion in non-phagocytic cells, and the ackA-pta operon encoding the acetate 
dissimilation system exhibited a low level of transcription during APEC infection. We also perform the in vitro adhesion and invasion assays by non-phagocytic (DF-1) cells, even hemagglutination (HA) assays. But this acetate assimilation system didn't take roles in APEC internalization (data not shown).

In higher eukaryotes, acetate plays a critical role in supporting energy homeostasis. Exogenous acetate produced by the intestinal commensal microbiota can be assimilated and used by various host metabolic pathways. Besides being a fuel for central metabolism, the emerging studies indicate that acetate might act as a metabolic signal in regulating host gene transcription [17, 49]. For example, acetate promotes the expression of genes, involved in fatty acid oxidation, to suppress body fat accumulation [57]. The effect of acetate assimilation system on host cell damage during APEC interaction with macrophages was assessed. Deletion of acs-yjcHactP operon in APEC significantly impaired its cytotoxic level to macrophages (HD11 and RAW264.7). Our study showed that acetate assimilation system promoted APEC to damage macrophages, when bacteria simultaneously replicated within macrophages. Recent research indicated that Group A Streptococcus (GAS) also simultaneously mediate its cytotoxic damage to human macrophages and cytosolic replication within macrophages, aiming to enhance GAS cell-to-cell spread [58]. The acetate assimilation system might be not only involved in APEC proliferation within macrophages but also promote be APEC evasion/reinfection of new macrophages.

Many pieces of evidences show that acetate produced by intestinal commensal microbiota has beneficial roles in host central energy metabolism and is closely correlated with multiple host physiological features. The acetate is the main component of intestinal short-chain fatty acids (SCFAs), which are the fermentation byproducts produced by the intestinal microbiota. The butyrate and propionate are the other major components of intestinal SCFAs, and three major SCFAs present about 60:25:15 of the molar ratio in the intestinal environment. The accumulating insights of intestinal SCFAs implicate that these byproducts play critical roles in the modulation of host energy metabolism, blood pressure, and inflammatory response, which is beneficial to host energy homeostasis and immunomodulatory functions [17]. However, for the pathogenic microorganisms, SCFAs can be used as an important induction signal, which modulate strongly the expression of virulence-related factors of intestinal pathogenic E. coli, such as Shigatoxigenic E. coli (STEC) O157:H7 strains. The SCFAs homeostasis (i.e., the steady concentration of SCFAs released by intestinal probiotics) in the intestinal environment might be out of balance by the response activity of pathogenic bacteria to deprive the intestinal microbiota-derived SCFAs [17]. As a typical example, the consumption imbalance of intestinal acetate caused by Vibrio cholerae leads to host metabolic disorders and lipid over-accumulation, eventually resulting in host lethality [59]. Liu et al. recently reported that SCFAs can suppress the production of proinflammatory cytokines in lipopolysaccharide (LPS)-stimulated macrophages through inhibiting LPS-induced NF- $\mathrm{kB}$ activation [38]. Our qRT-PCR results showed that the transcription of pro-inflammatory cytokines (IL-1 $\beta$, IL-6, IL-8, IL-12 $\beta$, and TNF- $\alpha$ ) and iNOS were obviously down-regulated in FY26 $\triangle a c s-y j c H$-actP infected macrophages compared to that in wild-type FY26 infected cells. An important incentive of the successful survival and proliferation during pathogenic bacteria interaction with phagocytosis is the excessive inflammation, accompanied by immune failure and body inflammatory damage [4]. Due to acetate involved in modulation of host inflammatory response $[17,49,51]$, our results revealed that the intracellular acetate consumption during facultative intracellular bacteria replication within macrophages might promote immunomodulatory disorders (i.e. imbalance of pro-inflammatory and anti-inflammatory responses), resulting in excessively pro-inflammatory responses of host macrophages. Intracellular acetate, besides serving as an energy source, exploited by APEC/ ExPEC during its proliferation in macrophages, as well as a signaling molecule, which was hijacked to avoid or suppress the immune response of macrophages. Whether or how intracellular acetate consumption leads to metabolic disorders of host macrophages will be determined to reveal more intimate host-microbe relationships.

APEC can enter the avian respiratory tract by dissemination of fecal dust, which is the major routine to infect avian extraintestinal niches $[44,52]$. When APEC colonizing avian lung tissues, macrophages play critical roles in host defenses to suppress APEC infection $[4,52]$. Since the acetate assimilation system encoded by acs-yjcHactP operon acted as a novel intracellular survival factor to promote APEC replication within macrophages, we further identified its roles in virulence-associated phenotype. Similar to survival in macrophages, the acetate assimilation system was also increasingly expressed during APEC colonization in the lung in vivo for avian colisepticemia models. Deletion of actP-yjcH-acs operon attenuated APEC virulence and colonization capability in avian lungs in vivo, suggesting that loss of this acetate system might impair APEC capability to pass through the immune defense of avian lung tissues. However, deletion of acs-yjcH-actP operon had no effect on the APEC virulence in murine sepsis model and bacteria proliferation in the avian blood in vivo. Our study indicated that the 
acetate assimilation system acted as a virulence factor and conferred a fitness advantage of APEC early colonization for avian colisepticemia models. Recent reports show that the macrophages of avian lungs fail to clear out highly virulent APEC, which can spread in the bloodstream to cause disease (i.e. avian colibacillosis) [13, 41]. Based on our research, the acetate assimilation system might facilitate APEC escape from phagocytes clearance of respiratory system and promote its entry the bloodstream to cause sepsis and multisystemic infection. Our research implies of the acetate permease (this outer membrane protein encoded by $a c t P$ gene) as a subunit vaccine candidate.

\section{Additional files}

Additional file 1. Bacterial strains and plasmids used in this study.

Additional file 2. Oligonucleotide sequences used as PCR primers.

\section{Abbreviations}

ExPEC: extra-intestinal pathogenic Escherichia coli; APEC: avian pathogenic Escherichia coli; Acs: acetyl-CoA synthetase; LDH: lactate dehydrogenase; NO: nitric oxide; iNOS: nitric oxide synthase; LB: Luria-Bertani; SCFAs: short-chain fatty acids; MOI: multiplicity of infection.

\section{Competing interests}

The authors declare that they have no competing interests.

\section{Authors' contributions}

$J D$ and $X Z$ conceived and designed the experiments; $X Z, Y S, M J$, and JW performed the experiments; XZ wrote the paper; $X Z, F X$, and JR analyzed the data; WZ offered suggestions; JD revised the manuscript and coordinated the research. All authors read and approved the final manuscript.

\section{Acknowledgements}

We thank Prof. Xiang Mao and Qinwei Sun for helpful advice, skillful technical assistance, and fruitful discussions.

\section{Author details}

${ }^{1}$ MOE Joint International Research Laboratory of Animal Health and Food Safety, College of Veterinary Medicine, Nanjing Agricultural University, Nanjing, China. ${ }^{2}$ Key Lab of Animal Bacteriology, Ministry of Agriculture, Nanjing Agricultural University, Nanjing 210095, China. ${ }^{3}$ China Pharmaceutical University, Nanjing 211198, China. ${ }^{4}$ Center for Post-doctoral Studies of Veterinary Medicine, College of Veterinary Medicine, Nanjing Agricultural University, Nanjing 210095, China. ${ }^{5}$ Center for Post-doctoral Studies of Animal Husbandry, College of Animal Science \& Technology, Nanjing Agricultural University, Nanjing 210095, China.

\section{Funding}

This work was supported by National Natural Science Foundation of China (Grant No. 31,672,576 and 31702252); National Basic Research Program of China (Project 973; 2015CB554200); and the Fund of Priority Academic Program Development of Jiangsu Higher Education Institutions (PAPD).

\section{Publisher's Note}

Springer Nature remains neutral with regard to jurisdictional claims in published maps and institutional affiliations.

Received: 16 December 2018 Accepted: 14 February 2019 Published online: 02 May 2019

\section{References}

1. Eisenreich W, Heesemann J, Rudel T, Goebel W (2015) Metabolic adaptations of intracellullar bacterial pathogens and their mammalian host cells during infection ("pathometabolism"). Microbiol Spectr 3:MBP-0002-MBP-2014

2. Kentner D, Martano G, Callon M, Chiquet P, Brodmann M, Burton O, Wahlander A, Nanni P, Delmotte N, Grossmann J, Limenitakis J, Schlapbach R, Kiefer P, Vorholt JA, Hiller S, Bumann D (2014) Shigella reroutes host cell central metabolism to obtain high-flux nutrient supply for vigorous intracellular growth. Proc Natl Acad Sci U S A 111:9929-9934

3. Chaston J, Goodrich-Blair H (2010) Common trends in mutualism revealed by model associations between invertebrates and bacteria. FEMS Microbiol Rev 34:41-58

4. Teng O, Ang CKE, Guan XL (2017) Macrophage-bacteria interactions-a lipid-centric relationship. Front Immunol 8:1836

5. Conover MS, Hadjifrangiskou M, Palermo JJ, Hibbing ME, Dodson KW, Hultgren SJ (2016) Metabolic requirements of Escherichia coli in intracellular bacterial communities during urinary tract infection pathogenesis. MBio 7:e00104-e00116

6. Lupp C, Robertson ML, Wickham ME, Sekirov I, Champion OL, Gaynor EC, Finlay BB (2007) Host-mediated inflammation disrupts the intestinal microbiota and promotes the overgrowth of Enterobacteriaceae. Cell Host Microbe 2:204

7. Czyz DM, Willett JW, Crosson S (2017) Brucella abortus induces a warburg shift in host metabolism that is linked to enhanced intracellular survival of the pathogen. J Bacteriol 199:e00227-e00317

8. Hackstadt T (1998) The diverse habitats of obligate intracellular parasites. Curr Opin Microbiol 1:82-87

9. Tala A, Progida C, De Stefano M, Cogli L, Spinosa MR, Bucci C, Alifano P (2008) The HrpB-HrpA two-partner secretion system is essential for intracellular survival of Neisseria meningitidis. Cell Microbiol 10:2461-2482

10. Creasey EA, Isberg RR (2012) The protein SdhA maintains the integrity of the Legionella-containing vacuole. Proc Natl Acad Sci USA 109:3481-3486

11. Ake FM, Joyet P, Deutscher J, Milohanic E (2011) Mutational analysis of glucose transport regulation and glucose-mediated virulence gene repression in Listeria monocytogenes. Mol Microbiol 81:274-293

12. Sharma O, O'Seaghdha M, Velarde JJ, Wessels MR (2016) NAD+-glycohydrolase promotes intracellular survival of group A Streptococcus. PLoS Pathog 12:e1005468

13. Zhuge X, Tang F, Zhu H, Mao X, Wang S, Wu Z, Lu C, Dai J, Fan H (2016) AutA and AutR, two novel global transcriptional regulators, facilitate avian pathogenic Escherichia coli infection. Sci Rep 6:25085

14. Cole JN, Nizet V (2016) Bacterial evasion of host antimicrobial peptide defenses. Microbiol Spectr 4:0006-2015

15. Setta A, Barrow PA, Kaiser P, Jones MA (2012) Immune dynamics following infection of avian macrophages and epithelial cells with typhoidal and non-typhoidal Salmonella enterica serovars; bacterial invasion and persistence, nitric oxide and oxygen production, differential host gene expression, NF-kappaB signalling and cell cytotoxicity. Vet Immunol Immunopathol 146:212-224

16. Plovier H, Cani PD (2017) Microbial impact on host metabolism: opportunities for novel treatments of nutritional disorders? Microbiol Spectr 5:0002-2016

17. Bernal V, Castano-Cerezo S, Canovas M (2016) Acetate metabolism regulation in Escherichia coli: carbon overflow, pathogenicity, and beyond. Appl Microbiol Biotechnol 100:8985-9001

18. Wolfe AJ (2005) The acetate switch. Microbiol Mol Biol Rev 69:12-50

19. Koh A, De Vadder F, Kovatcheva-Datchary P, Backhed F (2016) From dietary fiber to host physiology: short-chain fatty acids as key bacterial metabolites. Cell 165:1332-1345

20. Gimenez R, Nunez MF, Badia J, Aguilar J, Baldoma L (2003) The gene yjcG, cotranscribed with the gene acs, encodes an acetate permease in Escherichia coli. J Bacteriol 185:6448-6455

21. Sa-Pessoa J, Paiva S, Ribas D, Silva IJ, Viegas SC, Arraiano CM, Casal M (2013) SATP (YaaH), a succinate-acetate transporter protein in Escherichia coli. Biochem J 454:585-595

22. Zhu Ge X, Jiang J, Pan Z, Hu L, Wang S, Wang H, Leung FC, Dai J, Fan H (2014) Comparative genomic analysis shows that avian pathogenic Escherichia coli isolate IMT5155 (O2:K1:H5; ST complex 95, ST140) shares 
close relationship with ST95 APEC O1:K1 and human EXPEC O18:K1 strains. PLoS One 9:e112048

23. Vigil PD, Wiles TJ, Engstrom MD, Prasov L, Mulvey MA, Mobley HL (2012) The repeat-in-toxin family member TosA mediates adherence of uropathogenic Escherichia coli and survival during bacteremia. Infect Immun 80:493-505

24. Croxen MA, Finlay BB (2010) Molecular mechanisms of Escherichia coli pathogenicity. Nat Rev Microbiol 8:26-38

25. Wang Y, Yi L, Cai Y, Zhao W, Ding C (2016) Isolation, phylogenetic group, drug resistance, biofilm formation, and adherence genes of Escherichia coli from poultry in central China. Poult Sci 95:2895-2901

26. Cai W, Wannemuehler Y, Dellanna G, Nicholson B, Barbieri NL, Kariyawasam S, Feng Y, Logue CM, Nolan LK, Li G (2013) A novel two-component signaling system facilitates uropathogenic Escherichia coli's ability to exploit abundant host metabolites. PLoS Pathog 9:e1003428

27. Ewers C, Li G, Wilking H, Kiessling S, Alt K, Antao EM, Laturnus C, Diehl I, Glodde S, Homeier T, Bohnke U, Steinruck H, Philipp HC, Wieler LH (2007) Avian pathogenic, uropathogenic, and newborn meningitiscausing Escherichia coli: how closely related are they? Int J Med Microbiol 297:163-176

28. Breland EJ, Eberly AR, Hadjifrangiskou M (2017) An overview of twocomponent signal transduction systems implicated in extra-intestinal pathogenic E. coli infections. Front Cell Infect Microbiol 7:162

29. Alteri CJ, Mobley HL (2015) Metabolism and fitness of urinary tract pathogens. Microbiol Spectr 3:MBP-0016-2015

30. Zhuge XSY, Xue F, Tang F, Ren J, Li D, Wang J, Jiang M, Dai J (2018) A novel PhoP/PhoQ regulation pathway modulates the survival of extraintestinal pathogenic Escherichia coli in macrophages. Front Immunol 9:788

31. Teng CH, Cai M, Shin S, Xie Y, Kim KJ, Khan NA, Di Cello F, Kim KS (2005) Escherichia coli K1 RS218 interacts with human brain microvascular endothelial cells via type 1 fimbria bacteria in the fimbriated state. Infect Immun 73:2923-2931

32. Datsenko KA, Wanner BL (2000) One-step inactivation of chromosomal genes in Escherichia coli K-12 using PCR products. Proc Natl Acad Sci U S A 97:6640-6645

33. Zhu-Ge XK, Pan ZH, Tang F, Mao X, Hu L, Wang SH, Xu B, Lu CP, Fan HJ, Dai JJ (2015) The effects of upaB deletion and the double/triple deletion of $u p a B$, aat $A$, and $a a t B$ genes on pathogenicity of avian pathogenic Escherichia coli. Appl Microbiol Biotechnol 99:10639-10654

34. Kansal R, Rasko DA, Sahl JW, Munson GP, Roy K, Luo Q, Sheikh A, Kuhne KJ, Fleckenstein JM (2013) Transcriptional modulation of enterotoxigenic Escherichia coli virulence genes in response to epithelial cel interactions. Infect Immun 81:259-270

35. Wang S, Niu C, Shi Z, Xia Y, Yaqoob M, Dai J, Lu C (2011) Effects of ibeA deletion on virulence and biofilm formation of avian pathogenic Escherichia coli. Infect Immun 79:279-287

36. Li G, Ewers C, Laturnus C, Diehl I, Alt K, Dai J, Antao EM, Schnetz K, Wieler LH (2008) Characterization of a yjjQ mutant of avian pathogenic Escherichia coli (APEC). Microbiology 154:1082-1093

37. Beeckman DS, Rothwell L, Kaiser P, Vanrompay DC (2010) Differential cytokine expression in Chlamydophila psittaci genotype A-, B- or D-infected chicken macrophages after exposure to Escherichia coli O2:K1 LPS. Dev Comp Immunol 34:812-820

38. Liu T, Li J, Liu Y, Xiao N, Suo H, Xie K, Yang C, Wu C (2012) Short-chain fatty acids suppress lipopolysaccharide-induced production of nitric oxide and proinflammatory cytokines through inhibition of NF-kappaB pathway in RAW264.7 cells. Inflammation 35:1676-1684

39. Lavric M, Maughan MN, Bliss TW, Dohms JE, Bencina D, Keeler CL Jr, Narat M (2008) Gene expression modulation in chicken macrophages exposed to Mycoplasma synoviae or Escherichia coli. Vet Microbiol 126:111-121

40. Bateman SL, Seed PC (2012) Epigenetic regulation of the nitrosative stress response and intracellular macrophage survival by extraintestinal pathogenic Escherichia coli. Mol Microbiol 83:908-925

41. Mavromatis CH, Bokil NJ, Totsika M, Kakkanat A, Schaale K, Cannistraci CV, Ryu T, Beatson SA, Ulett GC, Schembri MA, Sweet MJ, Ravasi T (2015) The co-transcriptome of uropathogenic Escherichia coli-infected mouse macrophages reveals new insights into host-pathogen interactions. Cell Microbiol 17:730-746
42. Bokil NJ, Totsika M, Carey AJ, Stacey KJ, Hancock V, Saunders BM, Ravasi T, Ulett GC, Schembri MA, Sweet MJ (2011) Intramacrophage survival of uropathogenic Escherichia coli: differences between diverse clinical isolates and between mouse and human macrophages. Immunobiology 216:1164-1171

43. O'Neill AM, Thurston TL, Holden DW (2016) Cytosolic replication of group A Streptococcus in human macrophages. MBio 7:e00020-e00036

44. Zhuge X, Wang S, Fan H, Pan Z, Ren J, Yi L, Meng Q, Yang X, Lu C, Dai J (2013) Characterization and functional analysis of AatB, a novel autotransporter adhesin and virulence factor of avian pathogenic Escherichia coli. Infect Immun 81:2437-2447

45. Antao EM, Ewers C, Gurlebeck D, Preisinger R, Homeier T, Li G, Wieler LH (2009) Signature-tagged mutagenesis in a chicken infection model leads to the identification of a novel avian pathogenic Escherichia coli fimbrial adhesin. PLoS One 4:e7796

46. Li G, Feng Y, Kariyawasam S, Tivendale KA, Wannemuehler Y, Zhou F, Logue CM, Miller CL, Nolan LK (2010) AatA is a novel autotransporter and virulence factor of avian pathogenic Escherichia coli. Infect Immun 78:898-906

47. Tivendale KA, Logue CM, Kariyawasam S, Jordan D, Hussein A, Li G, Wannemuehler Y, Nolan LK (2010) Avian-pathogenic Escherichia coli strains are similar to neonatal meningitis E. coli strains and are able to cause meningitis in the rat model of human disease. Infect Immun 78:3412-3419

48. Mavromatis CH, Bokil NJ, Totsika M, Kakkanat A, Schaale K, Cannistraci CV, Ryu T, Beatson SA, Ulett GC, Schembri MA, Sweet MJ, Ravasi T (2014) The co-transcriptome of uropathogenic Escherichia coli-infected mouse macrophages reveals new insights into host-pathogen interactions. Cell Microbiol 17:730-746

49. Shimazu T, Hirschey MD, Huang JY, Ho LT, Verdin E (2010) Acetate metabolism and aging: an emerging connection. Mech Ageing Dev 131:511-516

50. Wang S, Dai J, Meng Q, Han X, Han Y, Zhao Y, Yang D, Ding C, Yu S (2014) DotU expression is highly induced during in vivo infection and responsible for virulence and $\mathrm{Hcp} 1$ secretion in avian pathogenic Escherichia coli. Front Microbiol 5:58

51. Vinolo MA, Rodrigues HG, Nachbar RT, Curi R (2011) Regulation of inflammation by short chain fatty acids. Nutrients 3:858-876

52. Horn F, Correa AM, Barbieri NL, Glodde S, Weyrauch KD, Kaspers B, Driemeier D, Ewers C, Wieler LH (2012) Infections with avian pathogenic and fecal Escherichia coli strains display similar lung histopathology and macrophage apoptosis. PLoS One 7:e41031

53. Peng L, Matthijs MGR, Haagsman HP, Veldhuizen EJA (2018) Avian pathogenic Escherichia coli-induced activation of chicken macrophage HD11cells. Dev Comp Immunol 87:75-83

54. Mittal R, Sukumaran SK, Selvaraj SK, Wooster DG, Babu MM, Schreiber AD, Verbeek JS, Prasadarao NV (2010) Fcgamma receptor I alpha chain (CD64) expression in macrophages is critical for the onset of meningitis by Escherichia coli K1. PLoS Pathog 6:e1001203

55. Hartman TE, Wang Z, Jansen RS, Gardete S, Rhee KY (2017) Metabolic perspectives on persistence. Microbiol Spectr 5:0026-2016

56. Atack JM, Ibranovic I, Ong CL, Djoko KY, Chen NH, Vanden Hoven R, Jennings MP, Edwards JL, McEwan AG (2014) A role for lactate dehydrogenases in the survival of Neisseria gonorrhoeae in human polymorphonuclear leukocytes and cervical epithelial cells. J Infect Dis 210:1311-1318

57. Kondo T, Kishi M, Fushimi T, Kaga T (2009) Acetic acid upregulates the expression of genes for fatty acid oxidation enzymes in liver to suppress body fat accumulation. J Agric Food Chem 57:5982-5986

58. Bastiat-Sempe B, Love JF, Lomayesva N, Wessels MR (2014) Streptoly$\sin \mathrm{O}$ and NAD-glycohydrolase prevent phagolysosome acidification and promote group A Streptococcus survival in macrophages. MBio 5:e01690-e01714

59. Hang S, Purdy AE, Robins WP, Wang Z, Mandal M, Chang S, Mekalanos JJ, Watnick PI (2014) The acetate switch of an intestinal pathogen disrupts host insulin signaling and lipid metabolism. Cell Host Microbe 16:592-604 